INRA Prod. Anim., 2017, 30 (5), 465-478

\section{Élevage et loups en France : historique, bilan et pistes de solution}

\author{
M. MEURETI, L. GARDE ${ }^{2}$, C.-H. MOULIN ${ }^{1}$, M.-O. NOZIÈRES-PETIT ${ }^{1}$, M. VINCENT

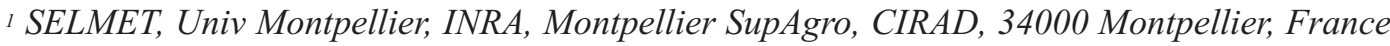 \\ ${ }_{2}$ CERPAM, 570 avenue de la Libération, 04100, Manosque, France \\ ${ }^{3}$ Écodéveloppement, INRA, 84914, Avignon, France
}

Courriel : michel.meuret@inra.fr

\begin{abstract}
La France a une politique élaborée de protection des troupeaux envers les loups. Pourtant, le nombre d'animaux domestiques tués, y compris dans des élevages protégés, se compte par milliers et augmente linéairement et rapidement depuis 2008. La dévaluation des moyens de protection résulte des capacités d'adaptation et du comportement opportuniste des loups, tirant parti de leur statut légal d'espèce sous protection stricte ${ }^{1}$.
\end{abstract}

Vingt-cinq ans après l'arrivée de loups depuis l'Italie au Parc National du Mercantour, 37 départements sont aujourd'hui concernés par leur présence, avec au total 57 zones de présence permanente, comportant 42 meutes (ONCFS 2017). Depuis 2002, le nombre d'animaux d'élevage prédatés, à savoir tués ou mortellement blessés, par des loups (ovins, caprins, bovins, équins...) se compte chaque année par milliers. En 2017, environ 12000 animaux prédatés ont été retrouvés, mais le total réel, disparus compris, avoisine plus probablement 15 000. Depuis 2008, la courbe de progression interannuelle du nombre d'animaux prédatés est linéaire. Ce à quoi il faut ajouter les autres dommages des attaques : stress à effet durable, avortements, blessures internes, baisse de fertilité, etc.

La France est pourtant le pays qui a mis en œuvre une politique de protection des troupeaux la plus élaborée d'Europe : chiens de protection, parcs de regroupement nocturne, présence humaine renforcée, notamment sur les estives de montagne. Comment interpréter la dévaluation rapide des moyens de protection, ainsi que la progression quasi ininterrompue du nombre d'animaux prédatés, qui conduit au sentiment, notamment chez les éleveurs, de perte de contrôle de la situation? C'est ce que nous tentons d'analyser ici.

Afin de faire comprendre le paradoxe auquel sont confrontés les éleveurs, nous revenons d'abord sur l'extension des zones de présence des loups en France, dans le cadre d'un projet conçu, documenté et suivi, tant politiquement que juridiquement, au niveau européen (European Commission 2017). Celui-ci prend à revers deux décennies d'efforts de mise en œuvre des politiques de l'agroenvironnement, visant à encourager les éleveurs à redéployer le pâturage pour contribuer à gérer des dynamiques de paysages devenus défavorables aux habitats de plusieurs centaines d'espèces de faune et de flore protégées. Or, du fait de l'abondance des lisières forestières, landes hautes et bosquets, le pâturage de ces milieux devient beaucoup trop risqué lorsque des loups y sont présents ou de passage.

Nous abordons ensuite les raisons de l'exposition à la prédation des élevages pastoraux et agropastoraux du Sud-est de la France, où $90 \%$ des pertes nationales restent aujourd'hui enregistrées. Nous décrivons les moyens de protection des troupeaux, dont l'adoption est généralisée dans le Sud-est, mais qui comportent autant d'avantages que d'inconvénients.

Enfin, nous analysons ce qui nous paraît être la principale raison de la dévaluation des moyens de protection : le manque d'anticipation, en France, du caractère particulièrement intelligent, adaptable et opportuniste des loups, ces derniers n'hésitant plus, ou peu, à privilégier les proies faciles d'élevage en l'absence de danger réel et clairement signifié. Les loups sont en effet arrivés en France sous statut légal de protection stricte, alors même que cet espace est occupé par de nombreux humains, la plupart inoffensifs, le braconnage y étant nettement moins développé qu'en Italie. Au fil des générations, ils se sont adaptés, n'associant apparemment plus le bétail aux humains et les humains au danger. C'est probablement la raison des attaques de plus en plus fréquentes à proximité des fermes, des routes et des villages, et aujourd'hui pour la moitié d'entre elles en cours de journée en Provence et dans les Alpes.

Une piste de solution consisterait à ré-inculquer aux loups la crainte des humains et de leurs activités d'élevage, en éliminant localement et promptement ceux qui s'en prennent aux troupeaux, qualifiés de « loups à problème " dans d'autres pays (Mech 2000). Ne pas exercer dans la durée une pression de sélection des loups sur la base de leur comportement de prédation, c'est admettre une profonde remise en question de la viabilité, vivabilité et reproductibilité des élevages pastoraux, agropastoraux, mais aussi herbagers.

\section{1 / Historique}

\section{1 / Une arrivée saluée comme une victoire}

De 1945 à 1989, 39 apparitions disparates de loups ont été recensées en France, dont un lâcher clandestin avéré de deux loups en 1968 dans les Landes (Campion-

\footnotetext{
${ }^{1}$ Cette synthèse sur la situation française s'inscrit dans les travaux plus larges du réseau de chercheurs COADAPHT, créé en juin 2016, et dont les produits sont accessibles à cette adresse : http://www.sad.inra.fr/Recherches/Coadaptation-predateurs-humains
} 
Vincent 2000). Un loup fut abattu en 1987 à Fontan (Alpes-Maritimes), dont le rapport d'autopsie signale qu'il était probablement issu de captivité (Pfeffer 1988). Deux autres observations datent de 1992, dans le Massif des Écrins et au Parc national du Mercantour. Cette dernière n'a été révélée au public qu'en 1993 par le magazine Terre Sauvage : "Nous avons choisi, avec les responsables $d u$ Parc [du Mercantour] et le Ministère de l'environnement, de garder le secret aussi longtemps que cela serait nécessaire à la sécurité des loups. Aujourd'hui, nous décidons de révéler leur présence : la clandestinité n'est pas un statut. » (Peillon et Carbonne 1993). "Le Mercantour, aujourd'hui, est en état de grâce. Le loup est revenu sans qu'il ait fallu se battre. [...] Avec son retour, la nature reprend vie. " (Adam 1993).

Dans le milieu des années 1990, et alors que la France ne comptait qu'une dizaine de loups, ceux-ci furent érigés en totem, au sens ethnologique du terme : ils procuraient à de nombreux militants une raison de se rallier à une cause d'apparence indiscutable. Le Président de France Nature Environnement (FNE) appela lui-même au combat: " Se battre pour un loup libre et sauvage, c'est se battre contre tous les enfermements, murs, prisons de toutes sortes, et contre l'appauvrissement de la biodiversité. [...] Sans ce super-prédateur, nos écosystèmes montagnards et forestiers s'abâtardissent, se changent en poulaillers à ciel ouvert. La vie a besoin du loup, l'homme de la vie. » (Brard 1996a). La notion de " poulailler à ciel ouvert » fut appréciée des éleveurs (Meuret et al 1997), notamment dans les AlpesMaritimes où ils subissaient déjà des pertes conséquentes en dépit de l'abondance des proies sauvages dans le secteur du Mercantour (figure 1).

Les propos du Président de FNE s'inscrivaient dans un projet de reconquête territoriale : «La collectivité nationale devra accepter la part du loup [avec présence de] 1000 à 1500 loups. » (Brard 1996b). Ce projet était, et encore, favorisé par le statut juridique très protecteur du loup (cf. encadré 1). En 1990, la France avait en effet mis en vigueur par décret la Convention de Berne relative à la conservation de la vie sauvage et du milieu naturel de l'Europe. Elle a surtout mis en ouvre en 1992 la Directive européenne Habitat-Faune-Flore, dite « Habitats », ayant pour objet la conservation des espèces remarquables et de leurs habitats, parmi lesquelles le loup.

Les premiers loups arrivent officiellement en France l'année suivante, sous statut de « protection stricte », bénéficiant ainsi de l'interdiction de toute forme de capture ou de mise à mort intentionnelle. Les textes de loi (cf. encadré 1)
Figure 1. Nombre de loups estimés de 1992 à 1996 (source : ONCFS) et nombre d'animaux d'élevage retrouvés tués ou mortellement blessés dans les Alpes Maritimes suite à des attaques de loups (source : DDT des Alpes Maritimes).

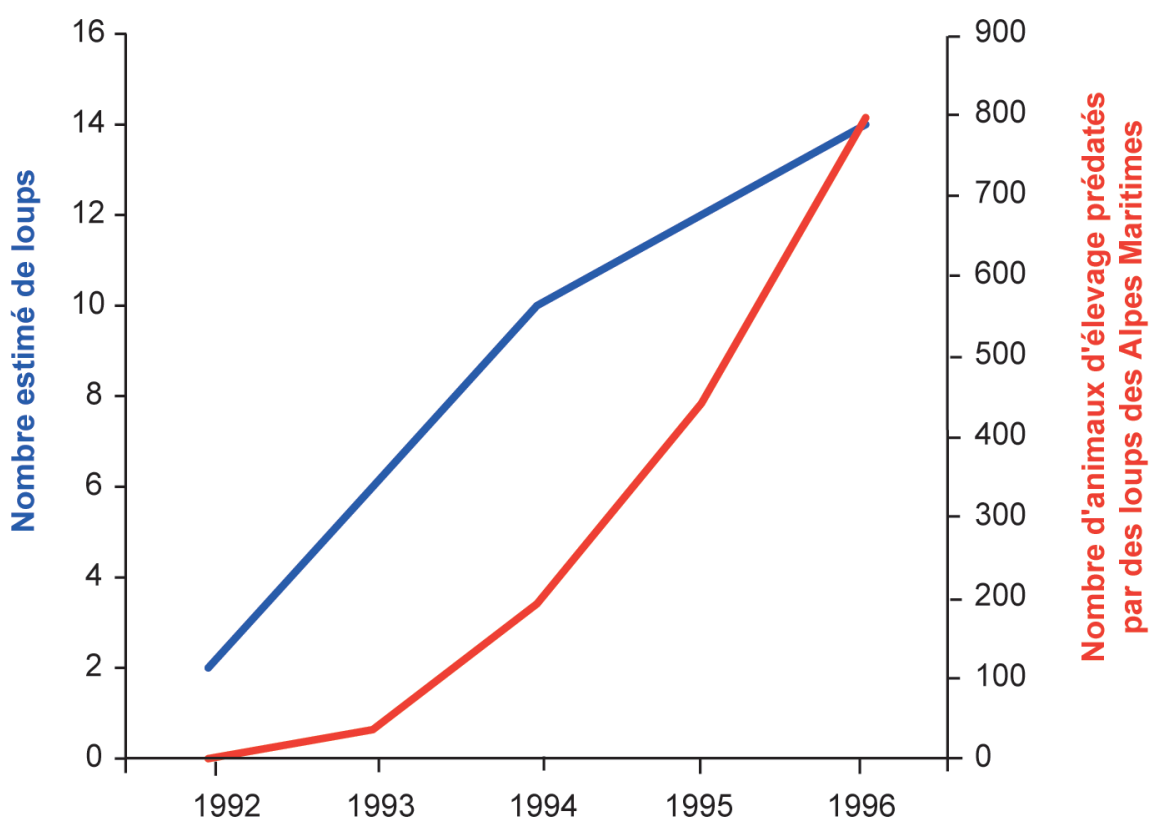

Années

Encadré 1. Le statut légal de protection du loup en France (source : Plan d'action national sur le loup 2013-2017, Dreal (Direction Régionale Environnement Aménagement Logement) Auvergne-Rhône-Alpes.

Le loup fait l'objet d'une protection stricte aux niveaux international, communautaire et national de par son inscription :

- à l'annexe II de la Convention de Berne relative à la conservation de la vie sauvage et du milieu naturel en Europe, ratifiée par la France en 1989 (loi 89-1004, décret $n^{\circ}$ 90-756 du 22 août 1990);

- aux annexes II et IV de la Directive européenne sur la conservation des habitats naturels ainsi que de la faune et de la flore sauvages (dite « Directive Habitats ») CEE 92/43 du 21 mai 1992 ;

- à l'annexe II de la CITES (Convention Internationale sur le Commerce des Espèces en Danger, 1973), ainsi qu'à l'annexe A de son règlement d'application européen ;

- sur la liste des mammifères protégés sur l'ensemble du territoire national, fixée par l'arrêté ministériel du 23 avril 2007. Les dispositions de la convention de Berne et de la directive Habitats sont par ailleurs transposées dans le code de l'environnement aux articles L.411-1, L.411-2 et R.411-1 à R.411-5.

Ces textes imposent que soient interdites toute forme de détention, de capture, de mise à mort intentionnelle, de perturbation intentionnelle, de commerce des spécimens prélevés dans la nature d'espèces de faune sauvage, parmi lesquelles le loup.

Des dérogations à ces interdictions peuvent intervenir à condition :

- que la dérogation ne nuise pas au maintien, dans un état de conservation favorable, des populations des espèces concernées dans leur aire de répartition naturelle ;

- que cette dérogation s'inscrive dans un cadre prédéfini, justifiant un intérêt à agir « pour prévenir des dommages importants à l'élevage » ;

- qu'il n'existe pas d'autre solution satisfaisante.

La destruction illégale d'un loup est punie en France de deux ans d'emprisonnement et de $150000 €$ d'amende. 
ouvrent néanmoins une possibilité de dérogation " afin de prévenir des dommages importants si la population [de loups] est en bon état de conservation et en l'absence de solutions alternatives satisfaisantes 》. Ces deux conditions méritent encore aujourd'hui d'être davantage documentées, tant biologiquement que techniquement. Nous y reviendrons.

\section{2 / L'élevage pastoral face à un paradoxe}

Depuis 1992, les concepteurs et opérateurs des politiques publiques européennes des paysages et de la nature se sont pour la plupart appuyés, en France, sur l'élevage pastoral et agropastoral pour accompagner leurs plans de gestion (Alphandéry et Billaud 1996, Pinton et al 2007). Au travers d'incitations financières contractualisées sur cinq ans les éleveurs ont été encouragés à reconquérir par le pâturage des espaces trop embroussaillés et parfois sujets au risque d'incendie (Hubert et al 2008). Aider à conserver ou restaurer des espaces de montagne, mais aussi de plaines ou de collines, avec mosaïques paysagères accueillant au total plusieurs centaines d'espèces remarquables et à protéger, devint une fonction reconnue de l'élevage. C'est dans ce contexte qu'une autre espèce remarquable, le loup, a fait irruption dans les Alpes.

Dès cette époque, comme le soulignait déjà Deverre (1999), les éleveurs se retrouvent face à un paradoxe : d'une part, ils sont encouragés au titre des dynamiques écologiques et de la prévention des incendies à redéployer le pâturage sur les espaces trop embroussaillés ; d'autre part, ils sont incités à se prémunir envers les loups, ces animaux attaquant souvent les troupeaux à partir de ces mêmes milieux, qualifiés de «paysages de la peur » en écologie (Laundré et al 2010). Ce paradoxe avait fait dire en 2002 à un gestionnaire de sites Natura 2000 dans les Alpes, nous ayant demandé de conserver son anonymat : " Le loup va nous faire perdre les bénéfices de dix ans de dialogue et négociations avec les éleveurs... Une espèce s'impose au détriment de plein d'autres. C'est sûr, on va perdre en capacité de gestion des habitats. »

\section{3 / La France au carrefour du projet de conservation des loups en Europe}

Pour restaurer ou mieux conserver des populations de grands carnivores (loups, ours bruns, lynx et gloutons), la Convention de Berne, puis la Directive Habitats de l'Union européenne, n'ont pas fixé ce qu'il faut entendre comme "état favorable de conservation des espèces 》. Chaque pays ayant une liberté d'interprétation, ceci génère difficultés et confusion lorsqu'il s'agit de suivre et d'éva- luer localement l'état des populations de loups (Trouwborst et al 2017).

Le « World Wildlife Fund» avait initié dès 1995 la constitution d'un groupe d'experts européens : la LCIE (Initiative pour les grands carnivores en Europe). Issus d'une vingtaine de pays et choisis intuitu personae pour leurs compétences personnelles, ces experts travaillent depuis 2010 sous l'égide de la Commission Survie des Espèces (SCS) de l'UICN (Union Internationale pour la Conservation de la Nature). Ils sont en contacts suivis avec les instances du Conseil de l'Europe et de la Commission Européenne. En appui sur des travaux scientifiques, la LCIE fait produire des synthèses sur les espèces et leurs états de conservation (LCIE 2017).

Les loups sont tout particulièrement suivis, du fait que leurs territoires sont

Figure 2. Lieux de présence permanente (rouge) ou occasionnelle (orange) des loups en Europe, ainsi que leurs 10 sous-populations recensées par les experts de la Commission européenne (d'après Boitani et al 2014).

Enveloppes de zonages colorés : notre interprétation des projets en cours au sein de l'UE visant à favoriser une meilleure conservation de l'espèce par des échanges entre certaines de ses sous-populations.

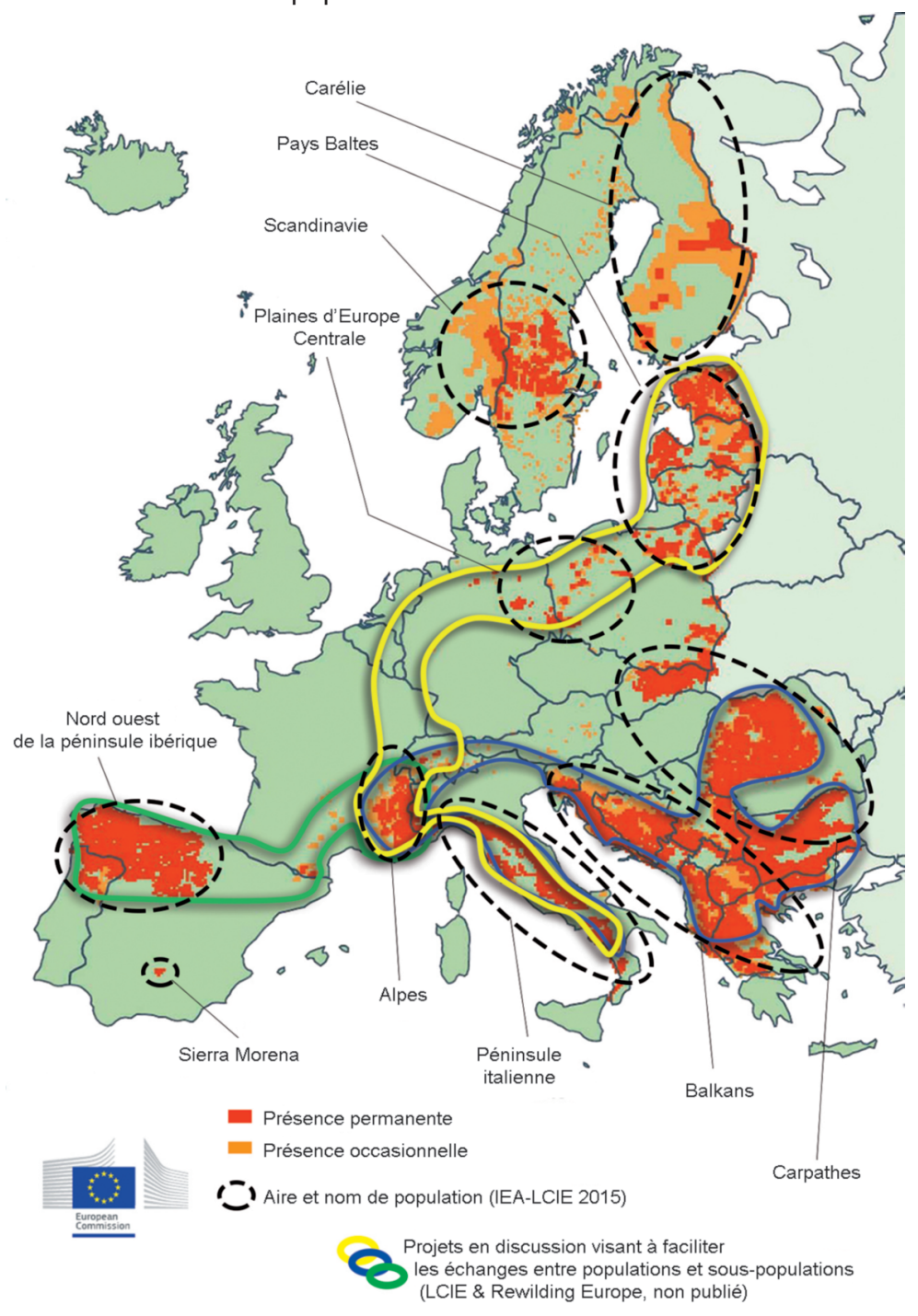


approche transfrontalière d'aspect moins directif au niveau de chaque pays qui a retenu l'attention de la Commission Européenne (Boitani et al 2014).

La LCIE dresse régulièrement des cartes de populations à l'échelle européenne. Pour ce qui concerne les loups, leur présence est aujourd'hui catégorisée en dix sous-populations (figure 2), avec comme enjeu majeur des corridors de connexion permettant de favoriser les échanges génétiques entre sous-populations. Posés comme nécessaires à la conservation à long terme, ils impliquent une politique transnationale des territoires et des effectifs qui a besoin, entre autres, de bases juridiques. Des experts s'y emploient, en appui notamment sur la jurisprudence de la Cour Européenne de Justice (Trouwborst 2010, Epstein et al 2016, Trouwborst et al 2017).

Tout l'est de la France, du Mercantour aux Vosges et à la Lorraine, se situe au carrefour de trois corridors escomptés (figure 2). Le Sud-est de la France tout particulièrement, avec l'étendue et la quasi contigüité de Parcs nationaux et régionaux, est un espace jugé très favorable à la dispersion des loups. Or, il se trouve que ces espaces sont aussi, et depuis des siècles, occupés et entretenus par l'élevage pastoral et agropastoral, qui a largement contribué à façonner la richesse biologique de ces paysages.

\section{2 / Les systèmes d'élevage concernés et leurs mesures de protection}

\section{1 / Des élevages très exposés à la prédation}

Dans le Sud-est de la France, région méditerranéenne et Alpes du Sud, les élevages d'herbivores en plein air sont particulièrement exposés à la prédation. Près d'un demi-million d'hectares de pâturages y sont tout ou partiellement embroussaillés et boisés (Dobremez et al 2016). Les éleveurs y trouvent des ressources fourragères à bon marché pour des troupeaux ayant appris à les consommer (Gautier et al 2006). La diversité de ces fourrages naturels permet d'assurer à la fois sécurité alimentaire et robustesse économique des élevages.

Depuis l'arrivée des loups, ces espaces sont devenus des zones à risque pour les troupeaux, la visibilité limitée permettant aux loups d'opérer leurs observations préalables et attaques bien plus discrètement que sur de grandes pelouses ou prairies non bordées de haies d'arbres et d'arbustes. De plus, dans ces régions, en raison de la quasi absence d'enneige- ment, les troupeaux sont menés au pâturage quasiment toute l'année, contre six à huit mois seulement dans les régions plus au nord ou plus en altitude.

Les modes de conduite des troupeaux (calendriers de reproduction et de pâturage) et choix de produits d'élevage (agneaux légers ou lourds, finis à l'herbe ou en bergerie...) exposent aussi de façon différenciée les troupeaux au risque de prédation. Ainsi, à l'échelle du territoire du Sud-est, le système d'élevage ovin dominant produit un agneau lourd, dont la croissance et la finition se déroulent en bergerie. Dans ce cas, ce sont essentiellement des brebis non allaitantes, vides ou en cours de gestation, ainsi que des agnelles, qui sont conduites sur les pâturages naturels, accidentés, aux ressources parfois éparses. Avec ces animaux dont la demande alimentaire reste relativement limitée, le berger peut pratiquer un gardiennage " serré » (conduite directive du troupeau au long de circuits quotidiens de pâturage ; Meuret et Provenza 2015b). En raison de la prédation, il ramène chaque soir le troupeau en parc de nuit ou en bergerie.

En revanche, les élevages producteurs d'agneaux « tardons » (ou « coureurs ») conduisent les mères et leurs agneaux ensemble au pâturage durant tout l'été et parfois l'automne selon les conditions de vente. La viande de ces agneaux, plus âgés et nourris à l'herbe, est appréciée des connaisseurs. Mais dans ce cas, les demandes alimentaires plus élevées pour la lactation des mères et la croissance des agneaux imposent de laisser le troupeau s'étaler davantage. Le berger pratique alors un gardiennage plus « souple » et distant. Ce type de troupeau a besoin de plus de temps en journée pour pâturer suffisamment. Son regroupement systématique de nuit en raison des prédateurs devient une contrainte particulièrement forte. Elle l'est davantage encore dans le cas de troupeaux laitiers, ovins ou caprins. Le système d'élevage ovin « tardonnier » est dominant dans les Alpes-Maritimes. Il est également très répandu dans le Var et les Alpes de Haute-Provence, à savoir dans les trois départements subissant les impacts de prédation les plus élevés à ce jour.

\section{2 / Des mesures de protection à contraintes multiples pour les éleveurs et bergers}

Dès 1997, des mesures de protection des troupeaux ont été encouragées par l'État français et financées sur fonds européens (Vincent 2011). Elles se sont ensuite généralisées (figure 3), avec l'appui des services pastoraux départementaux et sur la base de plans de financement nationaux successifs, jusqu'à l'actuel «Plan d'action national loup
2013-2017 " (Dreal Auvergne RhôneAlpes 2017). Issues de pratiques traditionnelles, elles avaient d'abord été testées aux Etats-Unis, en Italie et Suède (Mech 1995), à partir de techniques emboitées et fondées sur trois postulats relatifs au comportement du prédateur :

i) une présence humaine renforcée et continue auprès du troupeau suffit à tenir les loups à distance : l'aide-berger ;

ii) un obstacle supplémentaire, souvent plus attentif et vigilant que les humains, décourage les loups téméraires : les chiens de protection;

iii) un troupeau enfermé de nuit sous la garde des humains et des chiens ne subit plus d'attaque : le parc de nuit électrifié.

\section{a) L'aide-berger}

Au prix de coûts très significatifs en salaires, qui représentent plus de $70 \%$ du budget total des mesures de protection, ainsi que des contraintes de logement dans les cabanes de montagne conçues le plus souvent pour un unique occupant, l'aide-berger a une indéniable utilité. Son travail consiste à soulager le berger titulaire ou l'éleveur pour les tâches supplémentaires liées à la protection, mais aussi à prendre en charge rapidement les conséquences des attaques (repérage des animaux tués ou blessés, accueil des agents pour les constats...). La quantité de travail a été évaluée à $200 \mathrm{~h} /$ mois dans le cas d'un troupeau collectif de brebis en estive (Silhol et al 2007) et à $100 \mathrm{~h} /$ mois pour un troupeau individuel de brebis ou de chèvres hors estive (Garde et al 2007). Chez un éleveur individuel hors saison d'estive, ces temps de travaux supplémentaires se situent surtout en début de matinée, soirée et début de nuit. Cela représente donc une dégradation considérable de sa qualité de vie. L'aide d'un salarié pour un temps partiel sur des périodes éclatées dans la journée est d'autant plus difficile à mettre en œuvre que tous les éleveurs du voisinage ont les mêmes besoins aux mêmes heures, ce qui rend impossible une solution mutualisée par un collectif d'employeurs.

\section{b) Les chiens de protection}

Absents depuis plus d'un siècle des Alpes et du Massif Central, les chiens de protection, en majorité de race Montagnes des Pyrénées ou " patou », ont été introduits en urgence dans les troupeaux des Alpes françaises et de Provence à partir de la fin des années 1990. En 2016, 1755 chiens de protection étaient recensés par l'Administration en Région Provence-Alpes-Côte d'Azur. En l'absence de données accessibles pour la 
Figure 3. Nombres d'élevages ayant adopté en France les mesures de protection du troupeau envers les attaques de loups.

Source : DRAAF Rhône-Alpes.

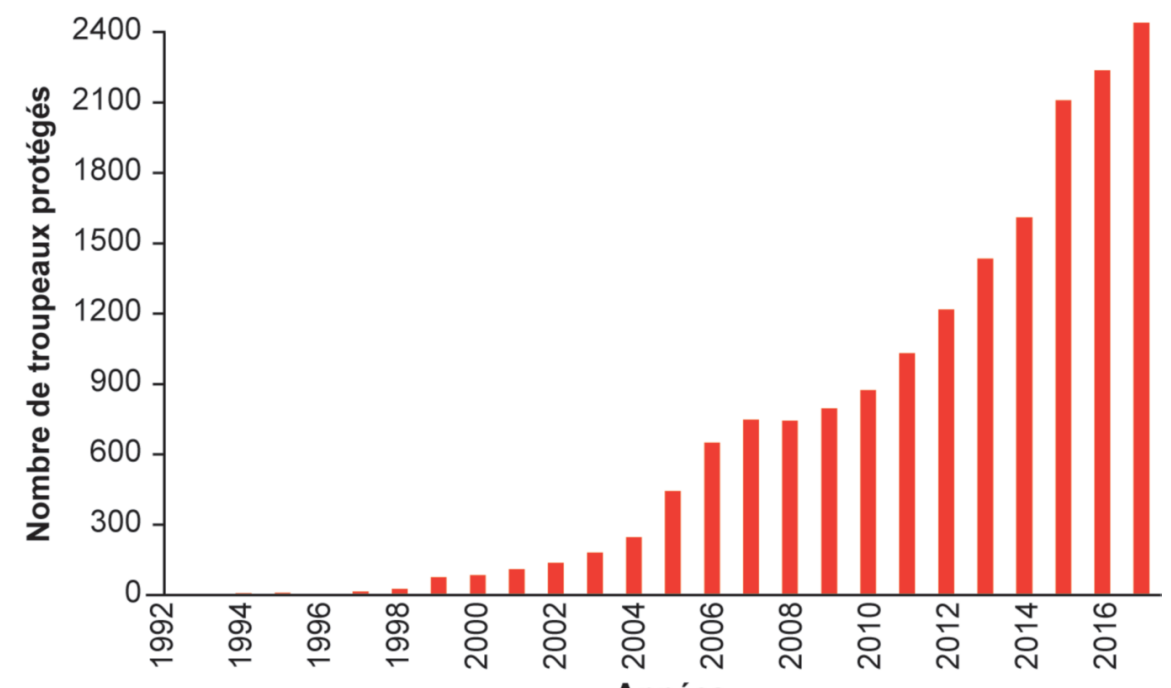

Région Auvergne-Rhône-Alpes, nous estimons à environ 3000 chiens l'effectif total présent actuellement en Provence et dans les Alpes.

Si la protection du troupeau par ces chiens peut, selon les cas, conduire à des résultats probants, elle génère en contrepartie de sérieuses difficultés. L'efficacité des chiens de protection commence avec un effectif minimum de deux chiens par troupeau, ou par lot d'animaux (Espuno 2004, Moret 2007). Cette efficacité est très variable, dépendant entre autres de : l'effectif et la distribution spatiale du troupeau au pâturage ; la nature du terrain pâturé ; la taille du parc de nuit ; l'effectif de loup(s) procédant à l'approche ou à l'attaque, organisés ou non en plusieurs groupes ; la connaissance préalable des loups envers ces chiens, mais aussi envers le terrain (Smith et al 2010). Un ratio d'un chien pour 200 brebis est souvent recommandé par les administrations en charge du dossier en France (de Roincé 2016). Dans les provinces italiennes des Abruzzes et de Molise, on trouve fréquemment 5 à 15 chiens pour des troupeaux de 500 à 2000 têtes, ainsi que des éleveurs qui insistent sur l'importance de travailler avec une meute de chiens, en tant que collectif stable et non divisible, face à une meute de loups (Garde 2016).

Les chiens de protection deviennent un souci majeur pour le multiusage de l'espace. Les conditions actuelles, en France, premier pays touristique d'Europe, exigent simultanément de la part de ces chiens de grandes réactivité et agressivité face aux loups, ainsi qu'une relative indifférence envers les humains nonéleveurs ou bergers qui fréquentent les mêmes lieux. Si nombre d'éleveurs trouvent leurs chiens trop placides face aux loups, la plupart des randonneurs
Années

les trouvent trop réactifs, pour ne pas dire agressifs à leur égard. Malgré les avertissements à visée pédagogique placés aux abords des sentiers, les plaintes se multiplient, des éleveurs passent devant les tribunaux suite à des morsures, et les maires sont de plus en plus nombreux à s'inquiéter de ce qui devient un problème de sécurité publique. Certains maires ont opté pour des avertissements inquiétants : "La commune décline toute responsabilité... »; d'autres, en zones très touristiques, restreignent ou interdisent le recours à ces chiens.

\section{c) Le parc de nuit électrifié}

Grands parcs de nuit électrifiés, permettant aux brebis ou aux chèvres de ne pas être trop entassées, doubles enceintes, proximité immédiate des chiens et des humains en alerte, la protection nocturne, lorsqu'elle est réalisable, peut s'avérer efficace. Néanmoins, cette troisième mesure, essentielle pour la protection, comporte aussi de gros inconvénients.

En alpage ou en collines, cette mesure fait obstacle à une bonne gestion pastorale, que ce soit en matière d'alimentation du troupeau, de renouvellement des ressources pâturées, ou de non érosion des sols (Vincent 2011). L'abandon de la pratique des « couchades libres », lieux de repos spontanément choisis par le troupeau, avec obligation de ramener chaque soir le troupeau dans le parc à côté de la cabane : i) polarise excessivement l'espace du territoire à pâturer ; ii) substitue des temps de déplacements non alimentaires à l'activité d'ingestion ; iii) augmente l'impact érosif suite aux passages répétés sur les pelouses ou les sols fragiles ; iv) a des conséquences néfastes sur l'état sanitaire des brebis, qui se retrouvent toutes les nuits dans des parcs devenus rapidement insalubres (Lapeyronie et Moret 2007).

L'obligation de ramener chaque soir le troupeau en parc de nuit désorganise profondément les possibilités d'ajustements des circuits de pâturage par un berger ou des enchaînements de parcs clôturés par un éleveur. Ces longs déplacements biquotidiens diminuent parfois de $20 \%$ le temps d'activité alimentaire au pâturage, surtout en périodes de forte chaleur, où les animaux mangent spontanément et plus confortablement « à la fraîche ". Les conséquences portent sur la motivation des animaux à prospecter l'espace, leur appétit, et en définitive leur état corporel et performances de production, surtout en fin de gestation et en lactation.

\section{3 / L'ampleur des dégâts en élevage}

\section{1 / La constante progression du nombre d'animaux prédatés}

Au cours des onze premières années (1994-2004), la progression du nombre d'animaux d'élevage prédatés, toutes espèces confondues, retrouvés et indemnisés suite aux attaques officiellement attribuées aux loups (figure 4) a été forte et régulière, jusqu'à approcher les 3000 animaux tués ou mortellement blessés par an. Durant cette période, l'adoption des contrats de protection par les éleveurs est restée limitée, avec moins de 300 troupeaux protégés (figure 3 ).

Durant cette période, des chiens divagants furent souvent accusés d'être à l'origine des attaques attribuées aux loups (Garde 1997). Des chiffres impressionnants furent avancés, affirmant que les dégâts de loups étaient peu de chose comparé aux " dizaines de milliers de bêtes » (Englebert 1998), voire même " plusieurs centaines de milliers " tuées chaque année par des chiens (Revet 2001, Wick 2003). Des enquêtes menées dans différentes régions avant l'arrivée des loups ont permis de mieux cerner cet impact des chiens. Les dégâts se sont avérés réels, mais très inférieurs aux chiffres annoncés, avec dans $89 \%$ des attaques, une identification et une élimination du chien en cause (Garde 2005, Brunschwig et al 2007, cf. encadré 2).

Entre 2004 et 2009, une nouvelle mesure financière initiée par le Ministère de l'Agriculture a permis de quadrupler le nombre d'éleveurs contractants. Ces derniers ont alors pu s'équiper massivement d'aides-bergers, de chiens de protection et de filets électrifiés pour les 
Figure 4. Évolution du nombre d'animaux d'élevage retrouvés tués ou mortellement blessés suite à des attaques officiellement attribuées à des loups (axe vertical de gauche - source : DDT(M) et DREAL Auvergne Rhône-Alpes ; données 2017 non stabilisées) et estimation du nombre de loups en France (axe vertical de droite source : ONCFS 2017).

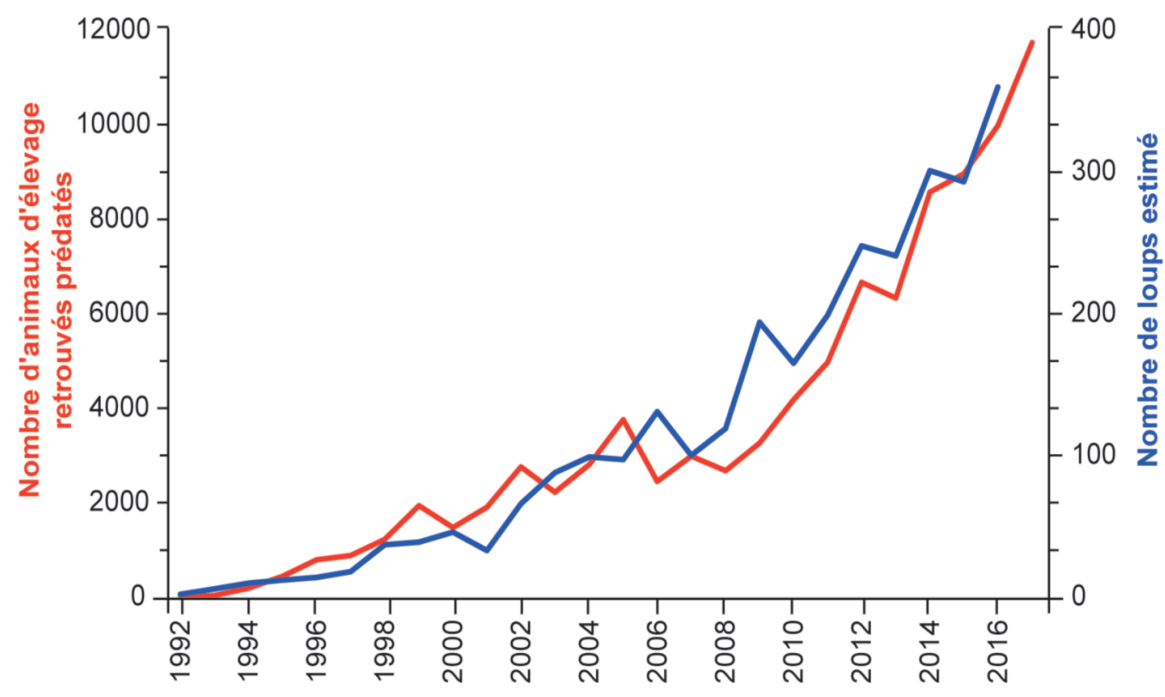

Encadré 2. Les chiens divagants et leur impact sur les troupeaux.

Contrairement à bien d'autres pays du monde, dont l'Italie et l'Espagne, les chiens ensauvagés, dits " errants » et sans maîtres, n'existent pas en France autrement que sous forme de rares individus à la durée de vie brève. Quant aux « divagants », à savoir les non surveillés, y compris les récemment abandonnés, les maires ont obligation de les faire saisir et mettre en fourrière (loi n999-5, J.O. du 7 janvier 1999).

Les attaques de chiens sur troupeaux ont toujours existé en France. Elles sont le fait de chiens du voisinage, parfois des chiens de chasseurs ou même d'éleveurs. La rurbanisation de nos campagnes est susceptible d'aggraver le phénomène, avec des propriétaires laissant leur chien seul et désœuvré sur un espace privé mais non efficacement clôturé (Bobbé 2000 ; Garde 2005).

Contrairement aux loups, les chiens sont aisément identifiables lors des attaques : ils ne cherchent pas à se dissimuler et se précipitent sur le bétail. Ils ne font généralement qu'une ou deux attaques avant d'être repérés, puis définitivement écartés si leur propriétaire refuse ou tarde à intervenir (Garde 2005 ; Garde et Meuret 2017).

Une enquête sur la prédation des ovins par des chiens hors zone de présence de loups a été réalisé (1999-2007) sur sept territoires d'élevage (Brunschwig et al. 2007) : Monges (Alpes de Haute-Provence), Lubéron oriental (Alpes de HauteProvence), Lubéron occidental (Vaucluse), Cévennes (Gard), Larzac (Aveyron), Livradois (Puy-de-Dôme), Jura (Doubs et Jura). Au total, 229 élevages furent enquêtés, soit 31 en moyenne par territoire. Le taux de prédation a été calculé en divisant le nombre total d'animaux prédatés durant la période d'enquête par le nombre total de ceux pâturant chaque année sur le territoire, ramené au nombre d'années de la période considérée. La fréquence d'attaques de chiens a été en moyenne de 0,20 , soit une attaque tous les cinq ans. Le taux annuel de prédation a été en moyenne de $0,26 \%$, soit seulement un animal prédaté par an pour un troupeau de 400 ovins. Chaque attaque de chien(s) a provoqué la perte de plusieurs ovins (de 1,6 à 22,9). Les attaques ont été en très grande majorité diurnes. Les chiens ainsi que leurs propriétaires ont été identifiés dans $89 \%$ des cas.

parcs de regroupement nocturne. Ce faisant, ils ont quasiment tous été obligés d'abandonner la pratique de « couchade libre » du troupeau. Cet investissement a apparemment stabilisé entre 2006 et 2009 le nombre d'animaux prédatés autour de 3000 par an. Cela suggérait une que le nombre d'éleveurs protégeant leur troupeau soit passé de 874 à 2440 (figure 3). Aujourd'hui, l'engagement des éleveurs dans les moyens de protection peut être considéré comme généralisé, car l'effectif de contrats correspond d'assez près au recensement du nombre d'Unités Pastorales (ou places de pâturage) dans les secteurs soumis à la prédation, en particulier sur les estives (Dobremez et al 2016).

Pour 2017, le nombre d'animaux retrouvés prédatés et à indemniser aux éleveurs atteindrait 11741 (figure 4), toutes espèces confondues : brebis, chèvres, veaux et génisses, chevaux et ânes. Ces chiffres ne tiennent pas compte des animaux disparus ou non indemnisables (non retrouvés sur terrain très accidenté, constats tardifs, consommés par les vautours, corbeaux ou renards), évalués d'après enquêtes de terrain à une bête manquante pour deux retrouvées (Bacha et al 2007). Le processus d'indemnisation des éleveurs prend actuellement en charge sur une base forfaitaire $20 \%$ d'animaux disparus suite aux attaques, en sus des animaux retrouvés morts ou mortellement blessés.

Comment expliquer la forte progression du nombre d'animaux prédatés depuis huit ans? Une première hypothèse est celle de la dynamique de colonisation des loups sur de nouveaux territoires, des départements jusqu'alors non concernés, où les éleveurs seraient mal ou peu préparés à protéger leurs troupeaux. Les loups ont en effet progressé significativement depuis 1993 (figure 5). Ils occupent à présent tout 1 'arc alpin ; ils ont aussi gagné le nord-est du pays, ainsi que le sud du massif central et l'est des Pyrénées. Au total, 37 départements sont aujourd'hui concernés par leur présence régulière ou irrégulière. En sortie d'hiver 2016-17, le Réseau Loup-Lynx de l'ONCFS comptabilise cinquante-sept «zones de présence permanentes » de loups, dont quarante-deux correspondent à des territoires de meutes (ONCFS 2017).

L'hypothèse selon laquelle c'est l'ampleur de la colonisation qui serait à l'origine de la forte progression du nombre d'animaux prédatés n'est pas validée. La figure 6 indique en effet que ce sont les éleveurs de sept départements initiaux, provençaux et alpins, qui subissent $90 \%$ des pertes totales. À lui seul, le département « d'arrivée des loups », les Alpes Maritimes, représentait encore 39\% des pertes totales en 2015 et $31 \%$ en 2016 . La forte hausse relative récente dans le Nord-est du pays ne concerne que 101 à 300 animaux prédatés.

certaine efficacité, y compris face à meutes de loups pour la plupart bien établies.

Toutefois, durant les huit années suivantes (2010-2017), la situation s'est brutalement dégradée, en dépit du fait
Une seconde hypothèse est celle d'attaques se déroulant sur des troupeaux non encore protégés. Elle ne tient également pas à l'épreuve des recensements des 
Figure 5. Présence de loup(s) détectée par période biennale sur l'exercice 2016 (données de mars 2013 à mars 2016) à l'échelle de mailles de 10 x $10 \mathrm{~km}$. Source des données : Réseau Loup-Lynx de l'ONCFS.

La présence du loup est appréciée selon un critère de quantité d'indices, y compris les attaques sur troupeaux. Une maille est classée en présence régulière si au moins 2 indices y ont été collectés durant chacune des deux dernières biennales considérées, sinon elle est classée en présence irrégulière.

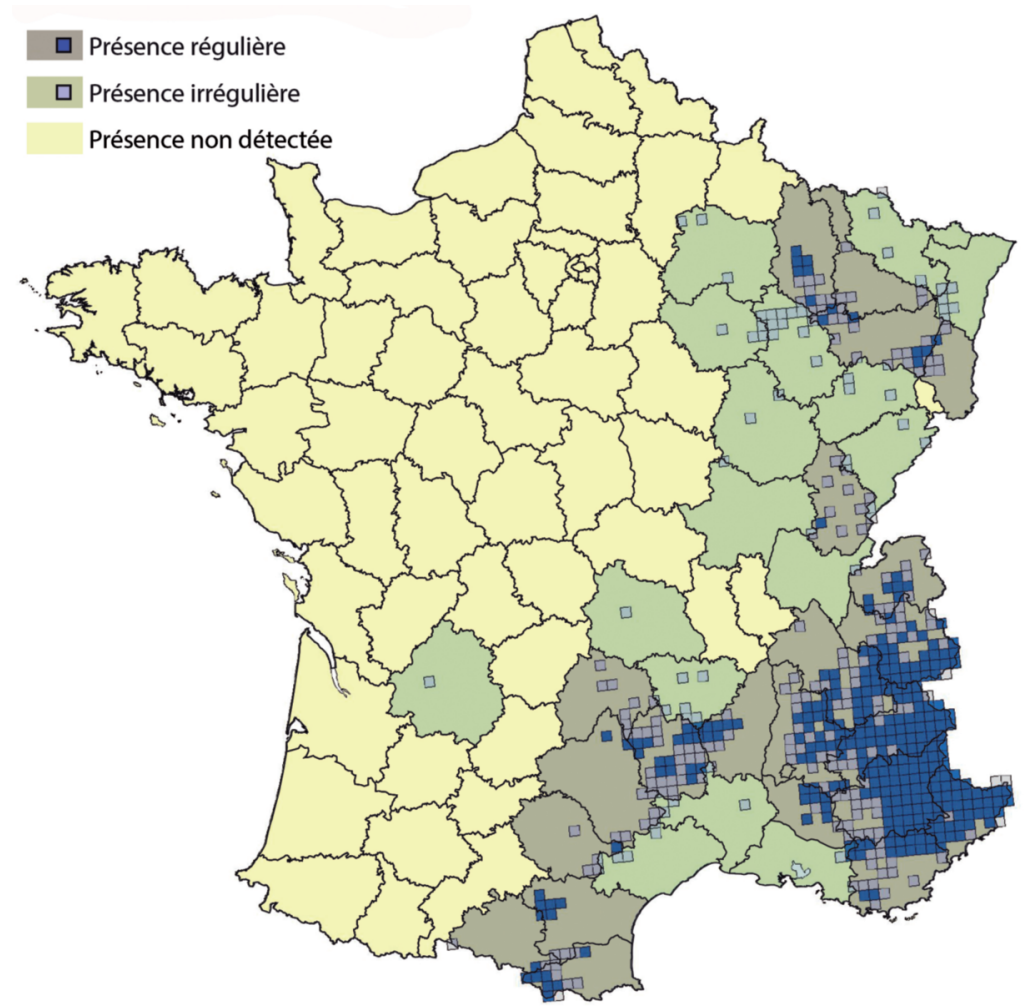

Figure 6. Nombre d'animaux d'élevage retrouvés tués ou mortellement blessés $d u$ fait de loup(s) par département en 2016.

Sources des données: DREAL et DRAAF Auvergne Rhône-Alpes, version au 25 avril 2017, données non stabilisées.

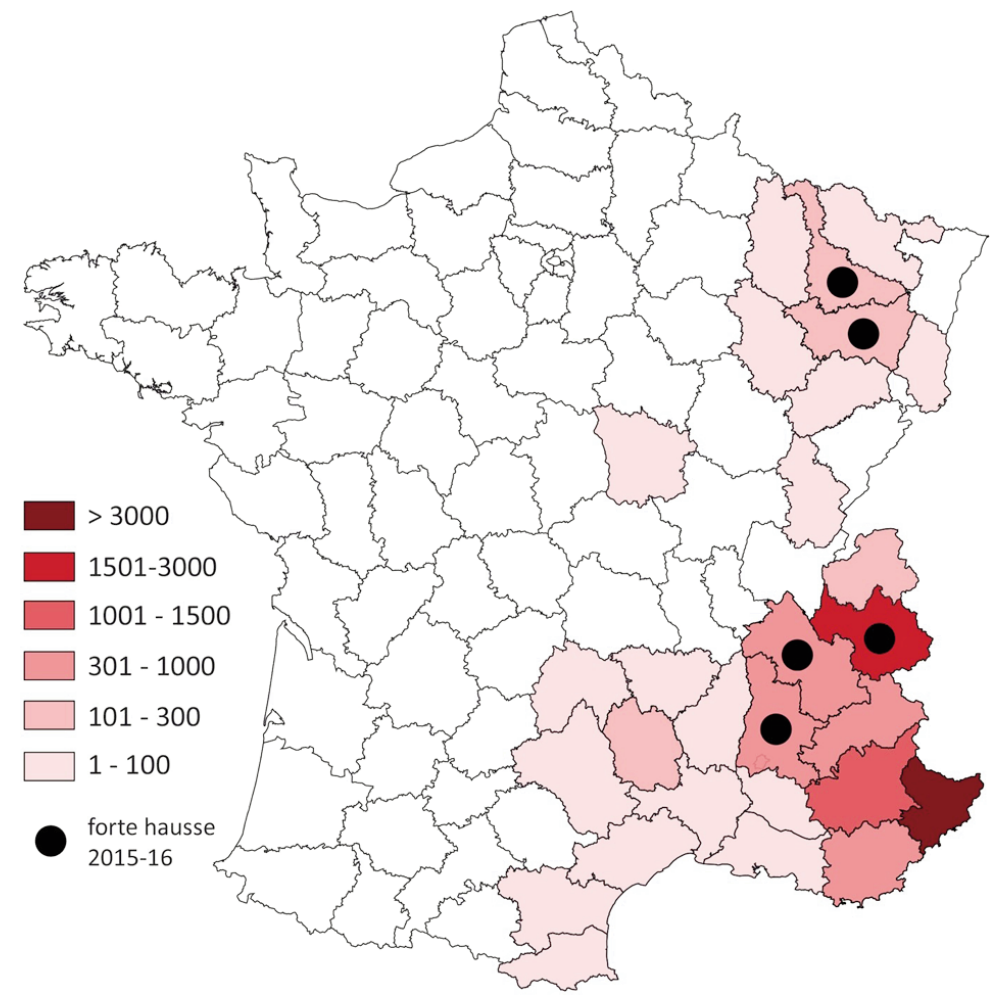

conditions d'attaques et du nombre d'animaux tués et mortellement blessés, tels que rassemblés par les administrations en charge du dossier. En effet, en Provence et dans les Alpes, les animaux tués ou mortellement blessés sont très majoritairement issus de troupeaux protégés (figure 7). C'était le cas en 2010 , et ce l'était encore davantage en 2015.

\section{2 / La diversification des proies domestiques}

Dès l'arrivée des loups en France, les éleveurs de chèvres ont également subi des pertes. On compte aujourd'hui 300 à 500 caprins tués par an (figure 8). Les bovins prédatés restent limités à une bonne centaine par an, mais ce nombre a toutefois doublé en trois ans. Les régions françaises où les loups sont aujourd'hui présents sont à forte majorité d'élevages ovins, ce qui contribue probablement à limiter les attaques sur bovins. Cela pourrait évoluer rapidement en cas de colonisation de nouvelles régions par les loups, notamment le Massif Central où domine l'élevage bovin en prairies.

Au nord de l'Espagne, au Portugal, ou dans les Montagnes Rocheuses des USA, régions d'élevage bovin majoritaire, les bovins tués par les loups sont tout aussi nombreux que les ovins (Iberian Pilot Action 2014, US Fish and Wildlife Service 2016). Quelques équins apparaissent depuis quelques années au tableau des animaux prédatés. Ceci provoque de l'inquiétude, aucune des mesures de protection n'étant adaptée à du bétail conduit au pâturage par lots d'effectif parfois très restreint (deux ou trois individus), dans des petits parcs clôturés, disséminés et distants. Quelques chiens, notamment de protection, sont aussi aujourd'hui devenus la proie des loups.

\section{3 / Les pertes dites « indirectes »}

Au-delà des animaux perdus ou disparus, l'état et les performances des troupeaux sont affectés de multiples autres manières, qualifiées de « pertes indirectes » par le Plan d'action national loup (Dreal Auvergne Rhône-Alpes 2017). La plupart des attaques provoquent une forte perturbation du troupeau, dont les conséquences sont particulièrement fortes lorsque les animaux sont en gestation ou en lactation. C'est pourquoi, est aujourd'hui indemnisé sur base forfaitaire le stress engendrant avortements, perte de poids, chute de lactation, ou baisses de fertilité. D'autres conséquences sont plus difficiles à déceler : blessures internes lorsque l'attaque a provoqué une panique en milieu escarpé ou dans un parc avec clôtures rigides.

Une autre conséquence non encore considérée mériterait de le devenir. Elle impacte fortement l'usage des pâturages 
Figure 7. Nombre d'animaux prédatés en 2010 et 2015, distinguant les troupeaux protégés des non protégés contre les loups en Régions PACA et AURA. Source : CERPAM - Base de données GéoLoup, DREAL Rhône-Alpes.

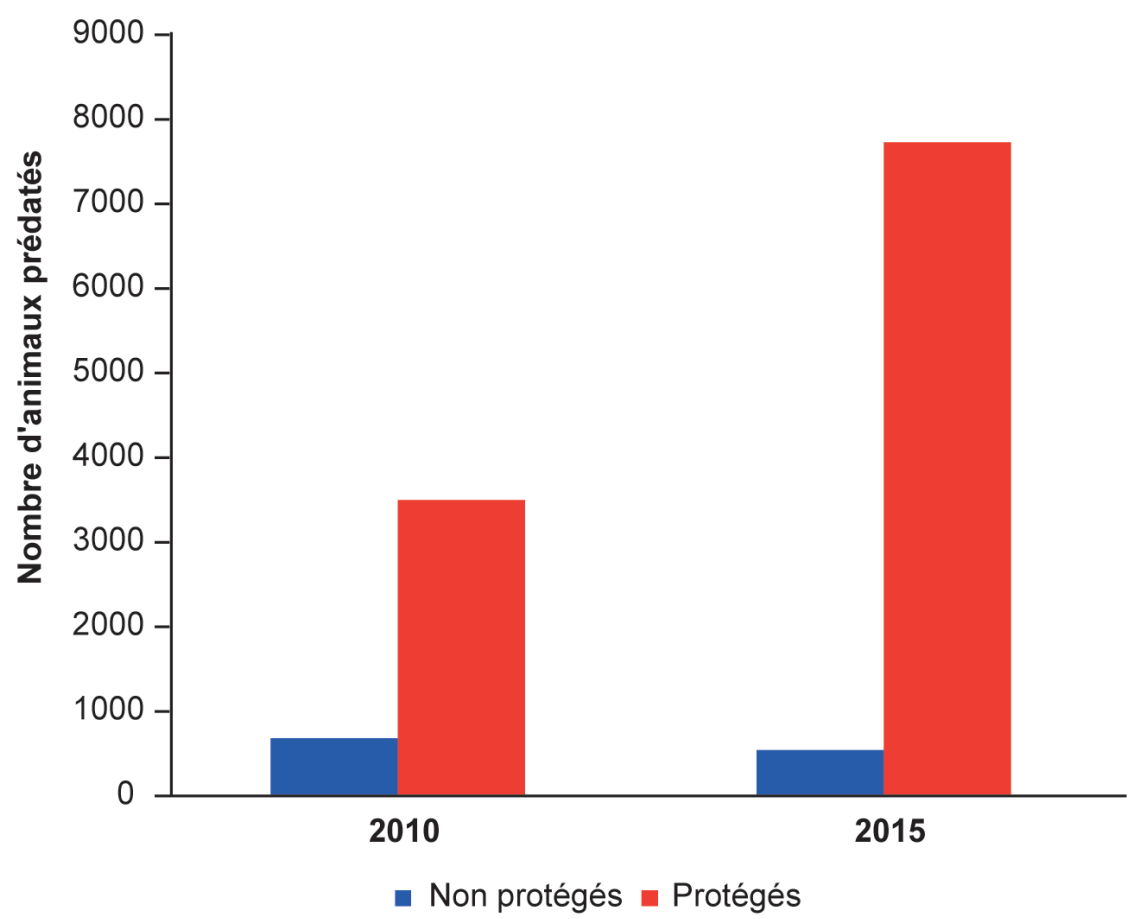

Figure 8. Nombre d'animaux retrouvés prédatés par des loups et classés selon les espèces.

Source : DREAL Auvergne-Rhône-Alpes, version au 25 avril 2017, données non stabilisées.

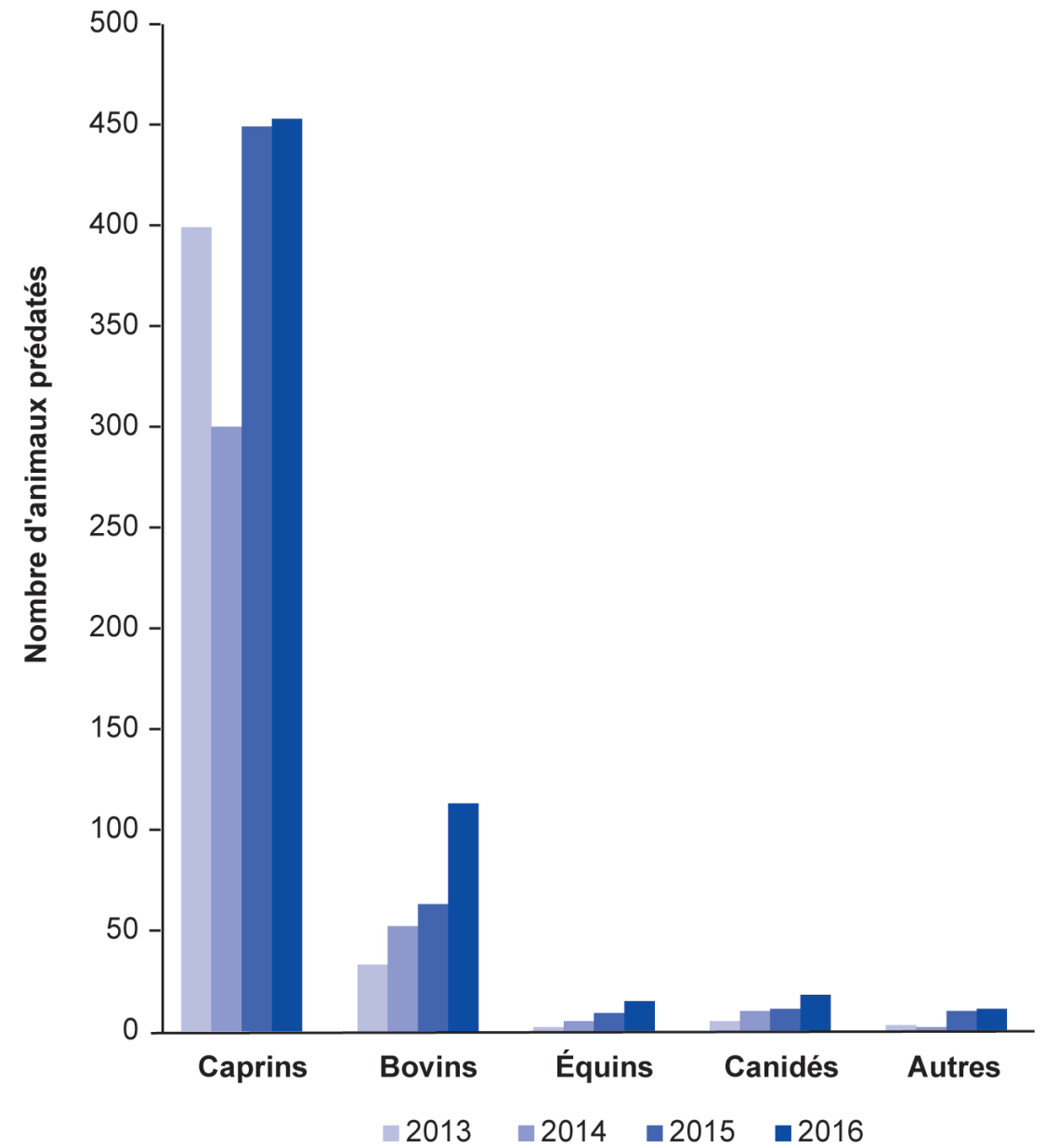

suite à des attaques de loups. Plusieurs éleveurs et bergers nous ont témoigné (Meuret et Provenza 2015a, Garde et Meuret 2017) à quel point il leur est difficile, et surtout très long (plusieurs semaines ou mois) de réussir à remettre en confiance un troupeau ayant été stressé lors d'une attaque de loups. C'est le cas avec des brebis, mais aussi avec des chèvres ou des vaches, qui mémorisent le lieu où s'est déroulé l'événement.

La mémoire des évènements survenus dans certains contextes et sur certains lieux est un processus étudié en neurosciences et sciences cognitives chez l'animal (Clayton et Dickinson 1998, Templer et Hampton 2013). Selon les éleveurs et bergers, cette mémoire persiste durant deux à trois ans (Meuret et Provenza 2015a). Avec persuasion, un berger est en mesure de reconduire le troupeau sur ces lieux, mais la plupart des animaux refuseront de s'y arrêter. Ce sont alors des surfaces de pâturage perdues, même si les ressources y sont abondantes et habituellement appréciées. Elles ne pourront être réutilisées qu'après deux ou trois ans, lorsque la mémoire de l'événement stressant se sera estompée, ou lorsque le troupeau sera largement renouvelé par des individus n'ayant pas mémoire de l'événement.

\section{4 / L'échec des mesures de protection : explication}

Comment expliquer l'échec des mesures de protection, malgré les sommes considérables engagées ? En $2016: 22,3$ millions d'euros alloués (part État) et 5,5 millions (part éleveurs) pour la protection ; 3,2 millions pour l'indemnisation des pertes. S'y ajoutent les salaires et les frais de fonctionnement des agents publics affectés au dossier du suivi des loups, des constats d'attaques et de leurs origines, l'accompagnement technique des éleveurs et le traitement administratif des dossiers, sans oublier la communication.

La France est le pays qui a mis en œuvre une politique de protection des troupeaux la plus élaborée d'Europe (voir 2.2.). Pourtant, c'est aussi la France qui, selon les rares statistiques disponibles et qui diffèrent en fiabilité selon les pays, subit les pertes en élevage apparemment les plus conséquentes dans le monde. Elles sont, par exemple, 30 fois supérieures à celles des trois États du nord des Rocheuses aux USA (Idaho, Wyoming et Montana), où l'élevage bovin et ovin est présent, y compris sur les estives, et les loups au nombre d'environ 1700 (US Fish and Wildlife Service 2016). Audelà de l'inévitable imperfection des mesures et des outils de protection, la 
raison principale de l'échec actuel provient selon nous des capacités des loups à s'adapter, tirant avantage en France de leur statut de protection stricte.

\section{1 / Les loups et leurs grandes capacités d'adaptation}

En matière d'écologie et éthologie du loup dans le monde, c'est l'ouvrage collectif de Mech et Boitani (2003) qui fait référence. Le loup y est décrit comme un animal très intelligent, doué d'une vie sociale en familles (meutes), donc apte aux apprentissages individuels et collectifs, particulièrement adaptable, très opportuniste, souvent imprévisible, et par ailleurs prolifique.

Les recherches sur les comportements de loups ont en très grande majorité été menées au Parc national de Yellowstone (États-Unis), ceci en raison des budgets considérables alloués là-bas depuis la réintroduction de loups canadiens en 1995-96 (Mech 2012). Cet espace de près de $9000 \mathrm{~km}^{2}$ a été préalablement vidé des Amérindiens, de toute autre activité agricole et de chasse, puis institué et géré en sanctuaire de nature (Pritchard 1999). Ce contexte particulier fait que les loups sont presque toujours envisagés, même implicitement, comme inféodés aux espaces sauvages. Or, à l'échelle mondiale, c'est loin d'être le cas (Lescureux et Linnell 2010a), et c'est d'ailleurs pourquoi ils ont acquis de telles capacités d'adaptation envers des environnements variés et changeants. Carnivores généralistes, et à l'occasion charognards, ils ont une alimentation très variée en proies sauvages : du bison au lièvre, en passant par les criquets, oiseaux, rongeurs, etc. (Peterson et Ciucci 2003). Cela leur a permis de survivre sur l'ensemble de l'hémisphère nord et d'y occuper la quasi-totalité des habitats (Mech 1995).

Sur tout l'hémisphère nord, les loups sont liés aux humains depuis plusieurs millénaires. Chasseurs de la préhistoire et loups occupaient vraisemblablement la même niche écologique, tous deux adaptés à la chasse en famille des grands herbivores (Fritts et al 2003). Ce n'est que dans l'histoire récente, et sur un espace restreint à l'Amérique du nord et à l'Europe, qu'ils ont été contraints de se réfugier en des lieux moins occupés. En France, jusqu'à la seconde moitié du 19 ième siècle, les loups étaient présents presque dans toutes les régions et mêlés, non sans conflits, au quotidien des habitants d'espaces ruraux alors densément peuplés (de Beaufort 1988, Moriceau 2007).

Les capacités d'adaptation des loups ne cessent de surprendre les scientifiques et de dérouter leurs modèles essayant de prévoir le développement spatial de leurs populations (Lescureux et Linnell 2010a). Par exemple, au nord-ouest de l'Espagne, les loups colonisent les plaines de grandes cultures végétales plutôt que les montagnes, alors que ces dernières leur sont a priori plus favorables du fait de la densité d'ongulés sauvages et de la couverture forestière (Blanco et Cortés 2007). Aux USA, les modèles prédictifs, tenant compte avant tout des qualités écologiques des habitats (Mladenoff et al 1999), ont été rapidement invalidés par les loups revenus occuper des territoires à faible probabilité de colonisation (Mech 2006).

Les loups s'adaptent donc aisément à la présence des humains et parviennent même à en profiter, que ce soit en s'attaquant à leur bétail vivant, aux cadavres d'animaux, ou en fouillant leurs déchets alimentaires (Peterson et Ciucci 2003). Depuis 70 ans, de nombreuses études ont cherché à caractériser les régimes alimentaires des loups à partir de leurs matières fécales, en y repérant la part occupée par les proies domestiques. Il en est conclu que les régimes sont aussi variés que le sont les habitats occupés (Lescureux et Linnell 2010a). Il en est conclu aussi que l'hypothèse selon laquelle il serait possible de prévoir la composition des régimes à partir des disponibilités relatives locales en proies sauvages et domestiques n'est pas toujours validée, les proies sauvages, même lorsqu'elles demeurent abondantes, n'étant pas nécessairement préférées aux domestiques (Anceau et al 2015, Miller 2015). Toutefois, des auteurs recommandent parfois encore la réintroduction d'ongulés sauvages afin de faire baisser la prédation en élevage (Meriggi et al 2014, Imbert et al 2016).

Figure 9. Nombre d'animaux d'élevage prédatés par des loups dans les départements alpins selon que les attaques se sont déroulées de nuit ou de jour. Source : Base GéoLoup, DREAL Auvergne-Rhône-Alpes ; traitement : CERPAM.

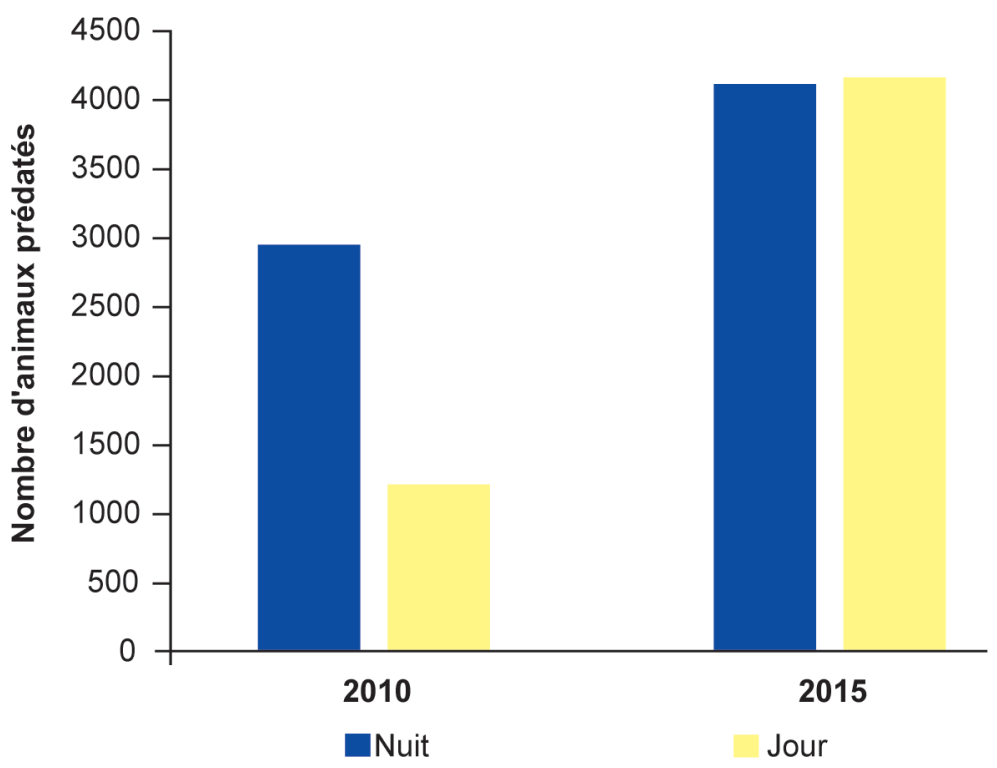

Les proies ne peuvent être résumées à des stocks relatifs plus ou moins abondants, puisque toutes apprennent à adopter des comportements de défense. Les proies préférées des loups sont celles qui tentent de leur échapper en fuyant ou en se dispersant, ce qui est typique des ovins et caprins, plutôt qu'en se regroupant et en leur faisant face (Mech et Peterson 2003). Les loups sont aussi stimulés par les mouvements de leurs proies, surtout lorsqu'elles sont nombreuses et groupées (Mech et al 2015). C'est peutêtre aussi la raison de l'attractivité des ovins et caprins domestiques, y compris lorsqu'ils sont regroupés en parcs de nuit et rendus affolés par la présence de face à des ongulés sauvages, les loups sont habitués à avoir un succès de chasse limité : 10 à 50\% (Mech et Peterson 2003). Ceci d'autant plus que leurs proies adoptent des comportements d'évitement, allant se réfugier sur des espaces moins accessibles (ex. barres rocheuses ou lits de cours d'eau). Par comparaison, les ongulés domestiques sont qualifiés de «proies faciles » par les écologues : leurs localisation et horaires de présence sont prévisibles ; ils ne peuvent se dissimuler, ni même se disperser ou s'enfuir, notamment lorsqu'ils sont conduits au pâturage en parcs clôturés.

En matière de capacité d'adaptation des loups en France, un exemple parmi les plus significatifs est l'actuel report des attaques en cours de journée, face à des troupeaux à présent regroupés dans leur quasi-totalité en parcs de nuit et sous la garde des chiens et des humains (figure 9). Autre signe d'adaptation : les attaques se déroulent toujours sur les estives de montagne, mais il y a de plus en plus fréquemment des attaques dans loups aux abords. Il faut souligner que, 
les vallées et les plaines, quasiment en toutes saisons dans les régions sans neige trop abondante. Parquer un lot d'animaux à proximité immédiate de la ferme ou du village n'est plus synonyme de tranquillité pour les éleveurs (figure 10).

\section{2 / En France, l'adaptation des loups était prévisible}

Comme dans bien des cas d'autres espèces dans le monde bénéficiant du statut de stricte protection (ours, lions, rapaces...), cette adaptation des loups est naturelle. Prévisible, elle n'a pourtant pas été anticipée en France.

"Le loup craint l'Homme " est une affirmation répétée, qui se fonde sur les expériences issues de pays où les loups sont abondants : Canada, Russie, Scandinavie, etc (Linnell et al 2002). Dans ces pays, les loups ont toujours été activement « régulés », surtout lorsqu'ils s'aventurent à proximité des humains et de leurs animaux domestiques. En Europe du sud, les loups protégés sont parfois fréquemment braconnés, comme par exemple en Italie où le nombre de loups braconnés a été évalué entre 200 et 300 par an (Galaverni et al 2015 ; Hindrikson et al 2016).

Quelle sorte d'humains le loup craintil ? Il craint l'équipe en séjour de chasse au trophée dans les Balkans ou en Russie. Il craint aussi, par exemple, le berger roumain employé en Italie, constamment vigilant et armé. Craint-il plus généralement tous les humains, y compris les nombreux et divers usagers de l'espace alpin français, randonneurs à pied, skieurs, vététistes et bergers, ces derniers uniquement équipés de bonnes chaussures et de jumelles? Imaginer cela, c'est nier au loup une part vitale de son intelligence : celle de réussir à identifier, par l'odorat, l'ouïe et la vue, les sources de danger réelles ou potentielles pour lui et sa progéniture.

En matière de protection des troupeaux, c'est le postulat qui fonde la première des trois mesures qui est erroné : « une présence humaine supplémentaire et continue auprès du troupeau suffit à tenir les loups à distance ». Ce postulat est validé dans tous les pays où les humains (éleveurs, bergers ou aides-berger, chasseurs) sont armés et autorisés à repousser activement les prédateurs menaçant le troupeau. Les prédateurs reconnaissent le bruit caractéristique du fusil qu'on charge, ainsi que l'odeur de la poudre, signaux interprétés comme une menace tangible et directe. Au Kirghizstan, des vêtements imprégnés d'odeur de poudre sont utilisés pour tenir les loups à distance, ceci pour autant que les humains procèdent à des « rappels » au sujet de la menace lui étant associée (Lescureux 2007).
Figure 10. Résultat d'une attaque nocturne de loup(s) le 4 mai 2017 (Savoie) sur un lot d'ovins arrivé la veille et placé en parc électrifié sous la garde d'un berger et de ses chiens : 23 agneaux et brebis tués (photo : Julien Morlot).

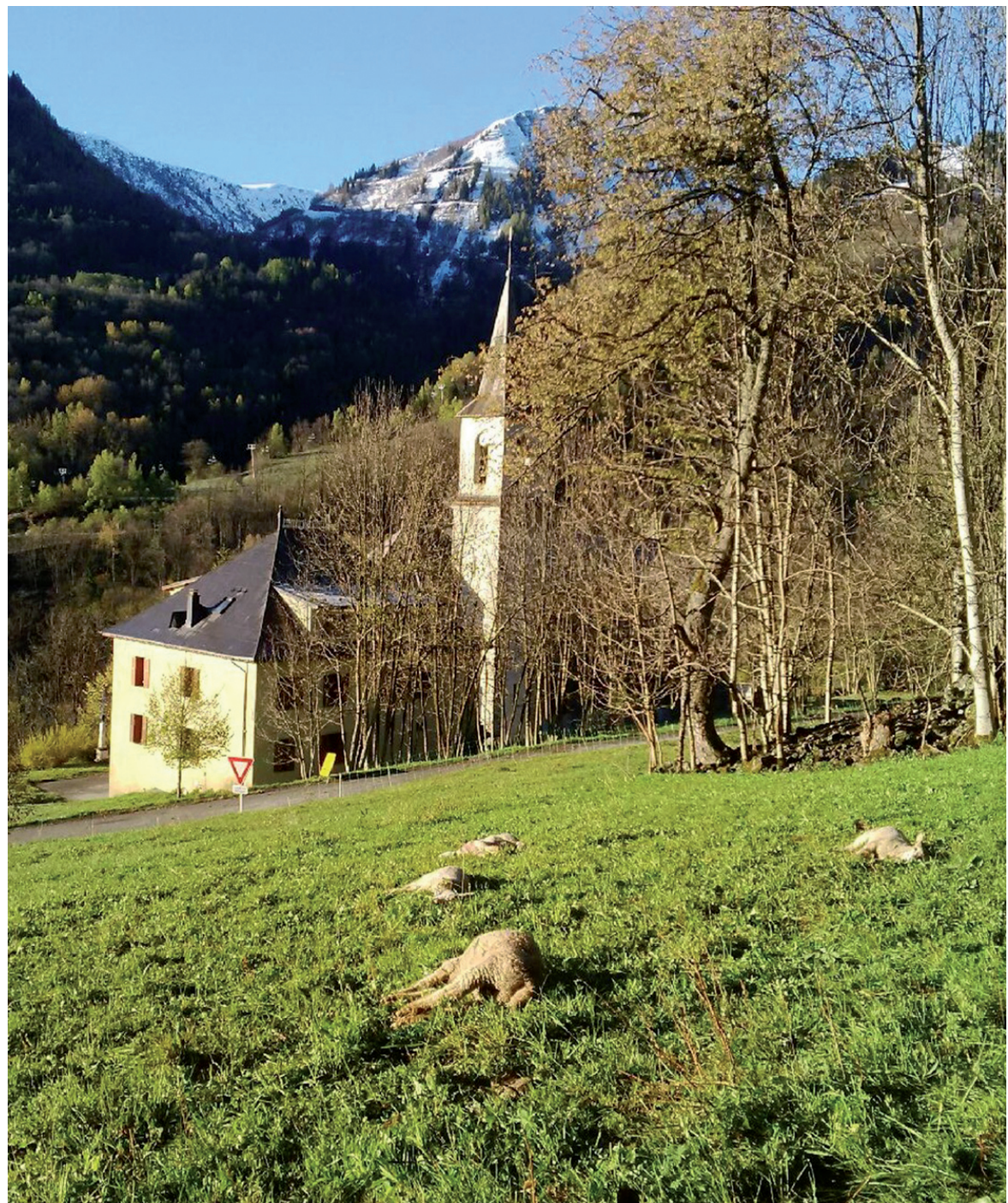

Les éleveurs et bergers en France, aussi motivés soient-ils, ne sont pas en mesure de signifier aux loups, à part avec quelques cris et gesticulations, qu'ils représentent une menace. Pour les loups, observant et jaugeant nos comportements avant d'agir, ce sont des humains inoffensifs parmi beaucoup d'autres, tels les randonneurs.

\section{5 / Pistes de solution}

Comment restaurer chez les loups arrivés en France un comportement d'animal sauvage, se tenant à distance des humains et de leurs activités ? Nous pouvons faire l'hypothèse que ces loups, après avoir été soumis en Italie à une pression de braconnage conséquente, ont en quelque sorte " trouvé refuge " chez nous, d'abord dans un Parc national, puis peu à peu ailleurs dans le pays où leur présence était nouvelle, inattendue, et la pratique du braconnage des loups bien moins développée que dans leur pays d'origine. Cela les aurait-il conduits à désapprendre la crainte des humains, et notamment celles des éleveurs et bergers ?

Par expérience ailleurs dans le monde, il apparaît qu'une des conditions à une coexistence entre humains et loups est l'établissement de relations de réciprocité, permettant de maintenir une distance acceptable (Lescureux 2007, Lescureux et al in press). La réciprocité peut notamment passer par des tirs et piégeages en cas d'attaques, ou velléités d'attaques sur troupeaux lorsque des loups sont vus à proximité, éliminant les individus ou les groupes les plus téméraires, jusqu'à parfois des meutes entières, l'objectif étant d'associer explicitement la présence d'humains et de leurs troupeaux à un danger (Bangs et al 2006, Lescureux et al in press).

Il est inutile de surenchérir dans les outils d'effarouchement : lampes clignotantes, rubans bicolores à poser le long des clôtures, drones mimant des aboiements de chiens, tonne-forts à gaz, fusées 
éclairantes, etc. Une revue des dizaines de techniques mises en œuvre depuis 1987 dans les Montagnes Rocheuses (USA) afin d'éloigner les loups des élevages aboutit à la conclusion que toutes, qu'elles soient visuelles, sonores ou olfactives, doivent être constamment associées à un danger immédiat. Sinon, après quelques jours, constatant qu'il n'y a aucune conséquence, les loups n'en tiennent plus compte (Bangs et al 2006).

Il en est de même pour ce qui concerne les chiens de protection. Leur capacité de vigilance (par l'odorat notamment) est très supérieure à celle des humains, mais ils ne sont efficaces que lorsqu'ils fonctionnent comme un « rappel » face à des loups craignant les humains parce qu'ils ont appris qu'il y a danger à s'en approcher. Avec des loups insistants, les chiens peuvent se faire déborder, surtout si le troupeau est étalé sur le pâturage, ou sur terrains accidentés lorsqu'il y a manque de visibilité. La solution de multiplier les chiens peut paraître intéressante, mais elle rend alors l'espace infréquentable aux autres usagers.

S'agirait-il d'armer les bergers, et surtout de les inciter à devenir constamment vigilants envers les prédateurs ? Cette solution est peu compatible avec le travail de garde du troupeau, qui doit privilégier le pilotage de l'alimentation et la maîtrise de l'impact des animaux sur les pâturages (Meuret 2010). Dans les pays où les bergers ou vachers font avant tout office de « vigiles », ils ne pilotent plus l'alimentation. Les animaux sont lâchés le matin hors du parc de nuit, surveillés à distance, puis regroupés et ramenés le soir (Ogada et al 2003, Barnes et Hibbard 2016). Quant à armer les aidesbergers, ceci exigerait une formation préalable spécifique, des compétences de tireur, et des salaires plus conséquents. Cela ne pourrait être envisagé que par binômes, puisque la vigilance constante et la réactivité immédiate sont exigées de jour comme de nuit.

Ré-inculquer la crainte des humains aux loups ne peut s'envisager que dans le cadre d'actions collectives à mener à l'échelle des territoires. Depuis bientôt trois ans, l'État français a pris conscience des limites de la protection des troupeaux par les seules mesures actuellement recommandées. Une brigade « loup » a été installée dans les Alpes à l'automne 2015, sous la responsabilité de l'ONCFS. Toutefois, même avec son effectif actuel de 15 agents, elle ne peut répondre aux sollicitations distantes et concomitantes. Cette initiative mériterait donc d'être développée ailleurs.

L'intervention de la brigade tend par principe à réaliser des « tirs ciblés 》 et localisés sur des loups s'attaquant à des troupeaux d'élevage, en renfort des éleveurs ayant déjà obtenu autorisation pour des tirs de défense de leur troupeau. Il est important, selon nous, de ne pas prétendre gérer par ces prélèvements l'effectif de sous-populations installées ou de passage en France. Il ne s'agit donc pas d'éliminer des loups, n'importe lesquels, au gré des rencontres inopinées avec des chasseurs de gibiers. Il s'agit d'éliminer de manière « ciblée » les loups qui, dans d'autres pays, sont qualifiés de « loups à problème », à savoir qui s'attaquent volontiers aux troupeaux en dépit des moyens de protection (Meuret 2011). Pour être efficace, cela impose un délai très court de réactivité sur site, ce qui est rendu difficile par l'actuelle procédure règlementaire d'autorisations de tirs de défense, et/ou d'interventions de la brigade, procédure visant aussi à se prémunir envers les recours judiciaires quasi systématiques intentés par des associations de défense des loups.

\section{Conclusion}

Les loups menacent la viabilité, la vivabilité et la reproductibilité des élevages organisés autour d'une conduite structurée par le pâturage sur ressources fourragères spontanées et cultivées. Or, l'immense majorité des conduites d'élevages en France s'appuie sur des lots de brebis, de chèvres ou de vaches distribués dans des parcs clôturés. Face aux loups, comment peut-on concevoir que toutes ces prairies, pelouses, landes et prés-bois soient à équiper de plus hautes clôtures électrifiées et de multiples chiens de protection? Sans oublier la surveillance humaine, si possible vigilante et armée, auprès de chaque lot.

Les moyens de protection des troupeaux, encadrés par une réglementation nationale structurée à partir des textes européens de la Convention de Berne et de la Directive Habitats, sont fondés sur le postulat selon lequel les loups ont peur des humains. Cette peur n'est en réalité pas un trait de comportement intrinsèque et permanent chez l'espèce. C'est un comportement acquis, et qui doit être constamment réinitié en associant explicitement la présence humaine rapprochée avec des risques réels pour les loups : blessures ou danger de mort. Les techniques d'effarouchement, ou répulsion non-létale, n'ont de sens que lorsqu'elles sont le signal de rappel du risque encouru en cas de non-respect. Par conséquent, techniques non-létales et létales doivent être mieux associées, ou du moins utilisables en chaîne et en cas de besoin (Bangs 2006, Lescureux et al in press). Traduit en langage administratif pour la France, cela impliquerait que des tirs de défense, ou des autorisations de piégeages à proximité des troupeaux attaqués, soient systématiquement accordés aux éleveurs et considérés au même titre que les actuelles mesures de protection.

Les travaux scientifiques qui permettraient de mieux informer ou de conforter les pistes de solutions gagneraient à privilégier l'investigation des savoirs et savoir-faire des acteurs de terrain dans différentes régions du monde. Plutôt que de simple coexistence, il s'agit d'un processus de coadaptation entre humains et loups, qui doit donc être étudié en conditions réelles et en n'omettant pas les effets de contexte (Lescureux et Linnell 2013, Garde et Meuret 2017, Mech 2017). Des recherches en écoéthologie des loups mériteraient d'être menées sur des terrains comportant des humains, ainsi que des proies sauvages et domestiques, puisqu'il est question de reports de prédation sur les unes ou sur les autres selon leurs comportements et les modes de protection de certaines proies. La mise à l'épreuve de techniques de protection gagne en pertinence lorsqu'elle est réalisée sur des territoires habituels de vie et de chasse du prédateur, ayant donc connaissance de tous les autres attracteurs ou répulseurs dans le paysage, mais aussi des comportements humains existants et pour la plupart prévisibles. $A$ contrario, des tests réalisés dans un contexte de parc zoologique impliquant d'un côté des loups nés en captivité et nourris régulièrement et de l'autre des attracteurs inertes (viande, animaux morts, artefacts) nous semblent d'une portée bien limitée. Il en est de même avec les tests comportementaux de chiens de protection. Leurs races sont nombreuses, mais leurs compétences et motivations à alerter et s'interposer dépendent de leurs habitudes acquises au sein de leur groupe social de naissance, sur un terrain et dans un contexte de travail particulier (Lescureux et Linnell 2014). La transmission des compétences au sein des groupes de chiens de protection, selon leurs conditions de vie et de travail en coordination avec des humains, est un thème de recherche prometteur.

En science, il devient urgent de traiter des capacités adaptatives des loups, mais aussi, et de concert, de celles des troupeaux et des humains qui en ont la responsabilité. Certaines formes de gestion adaptative en milieu réel mais soigneusement conçues peuvent être considérées comme des quasi-expérimentations (Williams et Brown 2014, Johnson et al 2015), avec des chercheurs privilégiant alors des enquêtes et suivis de terrains comparés. Vu l'étendue et la diversité des espaces déjà occupés par les loups dans le monde, les conditions à étudier sont nombreuses et riches d'enseignements 
lorsque les résultats sont rigoureusement contextualisés et présentés comme tels, sans excès de généralisation (Mech 2012, Allen et al 2017). Des savoirs et savoirfaire expérientiels existent. Ils peuvent être recueillis et leurs efficiences comparées par des approches relevant de l'ethnoécologie. Des situations intéressantes sont aussi celles de pays ou régions en transition, où des changements de réglementation et de pratique facilitent ou non la coadaptation avec les prédateurs (ex. Lescureux et Linnell 2013).

La généralisation de l'élevage en bâtiments sécurisés est une extrémité qui peut aussi s'envisager. Mais qu'en serat-il alors des produits d'élevage, du sens du métier pour de nombreux éleveurs, mais aussi des coûts de production, et donc de la viabilité des élevages, sans parler des dynamiques de paysages et de la qualité des habitats de centaines d'autres espèces sauvages à protéger ?

\section{Références}

Adam E., 1993. Éditorial. Terre Sauvage, 73, 4.

Allen B.L., Allen L.R., Andrén H., Ballard G., Boitani L., Engeman R.M., Fleming P.J.S., Ford A.T., Haswell P.M., Kowalczyk R., Linnell J.D.C., Mech L.D., Parker D.M., 2017. Can we save large carnivores without losing large carnivore science? Food Webs, http:// dx. doi.org/10.1016/j.fooweb.2017.02.008

Alphandéry P., Billaud J.P., 1996. Cultiver la nature. Études rurales, 141-142, 238p.

Anceau C., Bergeon J.P., Tardy X., Caratti G., Millisher G., Siméon D., Morand A., Loison A., Gaillard J.M., Houstin A., Duchamp C., Richard Q., Toïgo C., Maillard D., 2015. La prédation du loup sur les ongulés sauvages : impacts directs et indirects. Faune Sauvage, 306, 21-36.

Bacha S., Bataille J.F., Garde L., 2007. Indemnisation des pertes et évaluation des coûts réels. In : Loup - Elevage : s'ouvrir à la complexité. Actes du séminaire des 15 et 16 juin 2006. Garde L. (Éd). CERPAM, Manosque, France, 150-161.

Bangs E., Jimenez M., Niemeyer C., Fontaine J., Collinge M., Krsichke R., Handegard L., Shivik J., Sime C., Nadeau S., Mack C., Asher V., Stone S., 2006. Non-lethal and lethal tools to manage wolf-livestock conflict in the Northwestern United States. In: Proc. $22^{\text {nd }}$ Vertebrate Pest Conf., Timm R.M., O’Brien J.M. (Eds). Univ. California Davis publ., 7-16.

Barnes M., Hibbard W., 2016. Strategic grazing management using low-stress herding and night penning for animal impact. Stockmanship Journal, 57-71.

Beaufort (de) F.G., 1988. Écologie historique du loup, Canis lupus L. 1758, en France. Thèse d'État, Université de Rennes I, France, 1074p.

Blanco J.C., Cortés Y., 2007. Dispersal patterns, social structure and mortality of wolves living in agricultural habitats in Spain. J. Zool., 273, 114-124.

Bobbé S., 2000. Entre domestique et sauvage : le cas du chien errant. Une liminalite bien derangeante. Courr. Environ. INRA, 40, 66-74.

Boitani L., Alvarez F., Andren H., Avanzinelli E., Balys V., Blanco J.C., Breitenmoser U., Chapron G., Ciucci P., Dutsov A., Groff C., Huber D., Ionescu O., Knauer F., Kojola I., Kubala J., Kutal M., Linnell J., Majic A., Mannil P., Manz R., Marucco F., Melovski D., Molinari A., Norberg H., Nowak S., Ozolins J., Palazon S., Potocnik H., Quenette P.Y., Reinhardt I., Rigg R., Selva N., Sergiel A., Shkvyria M., Swenson J., Trajce A., Von Arx M., Wolfl M., Wotschikowsky U., Zlatanova D., 2014. Key actions for Large Carnivore popula- tions in Europe. Institute of Applied Ecology (Rome, Italy). Report to DG Environment European Commission, Brussels. Contract $\mathrm{n}^{\circ}$ 07.0307/2013/654446/SER/B3.

Brard L., 1996a. Joyeux Noël au loup des Alpes. Sci. Nature, 71,83 .

Brard L., 1996b. Manifeste pour un loup libre, vivant, sauvage, hors de toute idée de zonage barbelé. La Lettre du Hérisson, 177, 9-12.

Brunschwig G., Brosse-Genevet E., Dumontier A., Garde L., 2007. Dégats de chiens divagants et de prédateurs sauvages hors zone à loups resultats d'enquetes sur sept territoires d'elevage. Renc. Rech. Rum., 14, 165-168.

Campion-Vincent V., 2000. Les réactions au retour du loup en France: une analyse tentant de prendre " les rumeurs » au sérieux. Anthropozoologica, 32, 33-59.

Clayton N.S., Dickinson A., 1998. Episodiclike memory during cache recovery by scrub jays. Nature, 395, 272-274.

Deverre C., 1999. Le loup (le retour) et l'agneau (le départ ?). Courr. Envir. INRA, 36, 67-68.

Dobremez L., Bray F., Borg D., 2016. Principaux résultats de l'Enquête Pastorale 2012/2014 dan le massif des Alpes. IRSTEA, CERPAM, Réseau Pastoral Rhône-Alpes, ADEM, FAI, SEA 73 SEA 74, Suaci Montagn'Alpes, 81p.

Dreal Auvergne Rhône-Alpes, 2017. Plan d'action national loup 2013-2017, 52p. http://www auvergne-rhone-alpes.developpement-durable. gouv.fr/le-plan-d-action-national-loup-2013 2017-a3853.html

Englebert F., 1998. France Nature Environnement et la protection du loup. Info Loups, 4, 5.

Epstein Y., López-Bao J.V., Chapron G., 2016. A legal-ecological understanding of favorable conservation status for species in Europe Conserv. Letters, 9, 81-88.

Espuno N., 2004. Impact du loup (Canis lupus) sur les ongulés sauvages et domestiques dans le massif du Mercantour. Thèse doct., Universite Montpellier III, France, 214p.

European Commission, 2017. Large carnivore in the EU: the Commission's activity on large carnivores. http://ec.europa.eu/environment nature/conservation/species/carnivores/index en.htm

Fritts S.H., Stephenson R.O., Hayes R.D., Boitan L. 2003. Wolves and Humans. In: Wolves: behavior, ecology, and conservation, Mech L. D., Boitani L. (Eds). The Univ. of Chicago Press, Chicago, USA, 289-316.
Galaverni M., Caniglia R., Fabbri E, Milanesi P., Randi E., 2015. One, no one, or one hundred thousand: how many wolves are there currently in Italy ? Mammal Res., 61, 13-24.

Garde L., 1997. Loup et pastoralisme : la prédation et la protection des troupeaux dans la perspective de la présence du loup en Région Provence Alpes Côte d'Azur. Convention d'étude avec le Ministère de l'Environnement n ${ }^{\circ} 48 / 96$ du 30 août 1996 : 50p.

Garde L., 2005. Attaques de chiens sur les troupeaux ovins dans le Luberon et comparaison avec la prédation en territoires à loups. Anthropozool., $40,7-26$

Garde L., 2016. Molise et Abruzzes : des brebis, des chiens, des loups... et des Hommes. Pastum, $106,20-23$

Garde M., Meuret M., 2017. Quand les loups franchissent la lisière : expériences d'éleveurs, chasseurs et autres résidents de Seyne-les-Alpes confrontés aux loups. Rapp. CERPAM \& INRA Selmet, Montpellier, France, 116p.

Garde L., Bacha S., Bataille J.F., Gouty A.L., 2007. Les éleveurs résidents en zone à loups : perceptions et stratégies. In : Loup - Élevage : s'ouvrir à la complexité. Actes du séminaire des 15 et 16 juin 2006. Garde L. (Éd). CERPAM, Manosque, France, 180-191.

Gautier D., Guinamard C., Guérin G., Aussibal G., Beylier B., Garde L., 2006. Pâturer la broussaille : connaître et valoriser les principaux arbustes des parcours du Sud de la France. CERPAM, Manosque, France, 117p.

Hindrikson M., Remm J., Pilot M., Godinho R., Vik Stronen A., Baltrunaite L., Czarnomska S.D., Leonard J.A., Randi E., Nowak C., Akesson M., Lopez-Bao J.V., Alvares F., Llaneza L., Echegaray J., Vila C., Ozolins J., Rungis D., Aspi J., Paule L., Skrbinsek T., Saarma U., 2016. Wolf population genetics in Europe: a systematic review, meta-analysis and suggestions for conservation and management. Biol. Rev. Camb. Philos. Soc., 92, 1601-1629.

Hubert B., Meuret M., Bonnemaire J., 2008. Shepherds, sheep and forest fires: a reconception of grazingland management In: Handbook of transdisciplinary research. Hirsch Hadorn G. Hoffmann-Riem H., Biber-Klemm S., Grossenbacher-Mansuy W., Joye D., Pohl C., Wiesmann U., Zemp E. (Eds)., Springer, Berlin, Germany, 103-126.

Iberian Pilot Action, 2014. Exploring traditional husbandry methods to reduce wolf predation on free-ranging cattle in Portugal and Spain final report. Support to the European Commission's policy on large carnivores under the Habitats Directive - Phase two, Service contract $\mathrm{n}^{\circ}$ 07.0307/2013/654446/SER/B.3: 42p. 
Imbert C., Caniglia R., Fabbri E., Milanesi P., Randi E., Serafini M., Torretta E., Meriggi A., 2016. Why do wolves eat livestock? Factors influencing wolf diet in northern Italy. Biol. Conserv., 195, 156-168.

Johnson F.A., Eaton M.J., Williams J.H., Jensen G.H., Madsen J., 2015. Training conservation practitioners to be better decision makers. Sustainability, 7, 8354-8373.

Kaczensky P., Chapron M., Von Arx M., Huber D., Andrén H., Linnell J., 2013. Status, management and distribution of large carnivores - bear, lynx, wolf \& wolverine - in Europe, Update 2012. Large Carnivore Initiatiative for Europe, Report to EU Comm., Contract $n^{\circ} 070307201$ 2629085SERB3 : 72p.

Lapeyronie P., Moret A., 2007. Protection des troupeaux et impacts environnementaux. In : Loup - Élevage : s'ouvrir à la complexité. Actes du séminaire des 15 et 16 juin 2006. Garde L. (Éd). CERPAM, Manosque, France, 202-211.

Laundré J.W., Hernández L., Ripple W.J., 2010. The landscape of fear: ecological implications of being afraid. The Open Ecol. J., 3, 1-7.

LCIE, 2017. http://www.lcie.org/Publications ; http://www.medwolf.eu/index.php/cdpnews.html

Lescureux N., 2007. Maintenir la réciprocité pour mieux coexister? Ethnographie du récit Kirghiz des relations dynamiques entre les Hommes et les loups. Thèse doct. MNHN, Paris, France, 405p.

Lescureux N., Linnell J.D.C., 2010a. Les montagnes sont-elles les derniers refuges des grands prédateurs ? Histoire des Alpes, 15, 195-210.

Lescureux N., Linnell J.D.C., 2013. The effect of rapid social changes during post-communist transition on perceptions of the human - wolf relationships in Macedonia and Kyrgyzstan. Pastoralism, 3, 4. https://doi.org/10.1186/20417136-3-4

Lescureux N., Linnell J.D.C., 2014. Warring brothers: The complex interactions between wolves (Canis lupus) and dogs (Canis familiaris) in a conservation context. Biol. Conserv., 171, 232-245.

Lescureux N., Garde L., Meuret M., in press. Considering wolves as active agents to understand stakeholders' perceptions and develop management strategies. In: Large carnivore conservation and management: Human dimensions and governance, Hovardas T. (Ed). Routledge, Oxon, U.K.

Linnell J.D.C., Andersen R., Andersone Z., Balciauskas L., Blanco J.C., Boitani L., Brainerd S., Breitenmoser U., Kojola I., Liberg O., Løe J., Okarma H., Pedersen H.C., Promberger C., Sand H., Solberg E.J., Valdmann H., Wabakken P., 2002. The fear of wolves: a review of wolf attacks on humans. NINA, Trondheim, Oppdragsmelding, 731, 1-65.

Linnell J.D.C., Salvatori V., Boitani L., 2008. Guidelines for population level management plans for large carnivores in Europe. A Large Carnivore Initiative for Europe report prepared for the European Commission (Contract $\mathrm{n}^{\circ} 0705$ 01/2005/424162/MAR/B2), 85p.

Linnell J.D.C., Trouwborst A., Fleurke F.M., 2017. When is it acceptable to kill a strictly protected carnivore? Exploring the legal constraints on wildlife management within Europe's Bern Convention. Nature Conserv., 21, 129-157.
Mech L.D., 1995. The challenge and opportunity of recovering wolf populations. Biol. Conserv., 9, 270-278.

Mech L.D., 2000. The wolves of Minnesota: howl in the hearthland. Voyageur Press, Stillwater, MN, USA: 129 p.

Mech L.D., 2006. Prediction failure of a wolf landscape model. Wildl. Soc. Bull., 34, 874-877.

Mech L.D., 2012. Is science in danger of sanctifying the wolf? Biol. Conserv., 150, 143-149.

Mech L.D., 2017. Where can wolves live and how can we live with them? Biol. Conserv., 210, 310-317.

Mech L.D., Boitani L., 2003. Wolves: behavior, ecology, and conservation. The Univ. of Chicago Press, Chicago, USA, 448p.

Mech L.D., Peterson O., 2003. Wolf-prey relationships. In: Wolves: behavior, ecology, and conservation, Mech L.D., Boitani L. (Eds)., The Univ. of Chicago Press, Chicago, USA, 131-160.

Mech L.D., Smith D.W., MacNulty D.R., 2015. Wolves on the hunt: the behavior of wolves hunting wild preys. The Univ. of Chicago Press, USA, 187p.

Meriggi A., Dagradi V., Dondina O., Perversi M., Milanesi P., Lombardini M., Raviglione S., Repossi A., 2014. Short-term responses of wolf feeding habits to changes of wild and domestic ungulate abundance in Northern Italy. Ethol. Ecol. Evol., 27, 389-411.

Meuret M., 2010. Un savoir-faire de bergers Quae \& Educagri Eds., Versailles \& Dijon, France, 336p.

Meuret M., 2011. Peut-on vivre avec les loups en France : il faut une gestion ciblée des loups et des meutes à problèmes. La Recherche, 448, 102 .

Meuret M., Provenza F.D., 2015a. When Art and Science meet: integrating knowledge of French herders with science of foraging behavior. Rangel. Ecol. Manage., 68, 1-17.

Meuret M., Provenza F.D., 2015b. How French shepherds create meal sequences to stimulate intake and optimise use of forage diversity on rangeland, Anim. Prod. Sci., 54, 309-318.

Meuret M., Chabert J.P., Deverre C., 1997. Entre brebis et loups... Le Sadoscope, 90 (suppl.) : 1-4

Miller J.R.B., 2015. Mapping attack hotspots to mitigate human-carnivore conflict: approaches and applications of spatial predation risk modeling. Biodiv. Conserv., 24, 2887-2911.

Mladenoff D.J., Sickley T.A., Wydeven A.P., 1999. Predicting gray wolf landscape recolonization: logistic regression models vs. new field data. Ecol. Appl., 9/1, 37-44.

Moret A., 2007. L'utilisation du chien de protection dans les Alpes françaises. In : Loup Élevage : s'ouvrir à la complexité. Actes du séminaire des 15 et 16 juin 2006. Garde L. (Éd). CERPAM, Manosque, France, 118-129.

Moriceau J.M., 2007. Histoire du méchant loup 3000 attaques sur l'homme en France (XVe-XXe siècle). Fayard, Paris, France, 640p.

Ogada M.O., Woodroffe R., Oguge N.O. Frank L.G., 2003. Limiting depredation by
African carnivores: the role of livestock husbandry. Conserv. Biol., 17, 1521-1530.

ONCFS, 2017. Bulletin d'information du réseau Loup-lynx, $36: 31 \mathrm{p}$

Peillon A., Carbonne G., 1993. Bienvenue aux loups. Terre Sauvage, 73, 23-42.

Peterson R.O., Ciucci P., 2003. The wolf as a carnivore. In: Wolves : behavior, ecology, and conservation. Mech L.D., Boitani L. (Eds)., The Univ. of Chicago Press, Chicago, USA: 104-130.

Pfeffer P., 1988. Lettre au sujet de l'examen post-mortem du loup de Fontan adressée au Parc National du Mercantour. 18 mars 1988. Présidence du Comité scientifique du Muséum National d'Histoire Naturelle, Paris, France, $1-2$.

Pinton F., Alphandéry P., Billaud J-P., Deverre C., Fortier A., Geniaux G., 2007. La construction du réseau Natura 2000 en France. La Documentation française, Coll. L'environnement en question, Paris, France, 249p.

Pritchard J.A., 1999. Preserving Yellowstone's natural conditions : science and the perception of nature. University of Nebraska Press, Lincoln, Nebraska, USA, 370p.

Revet P., 2001. Chiens errants : le grand carnage. Le Chasseur Français, Septembre 2001, 164-168.

Roincé (de) C., 2016. Évaluation de l'efficacité des moyens de protection des troupeaux domestiques contre la prédation exercée par le loup : période 2009-2014. TerrOïko, Rapport au Ministère de l'Agriculture, de l'Agroalimentaire et de la Forêt et Ministère de l'Environnement, de l'Énergie et de la Mer, 64p.

Silhol A., Bataille J.F., Dureau R., Garde L., Niez T., 2007. Evaluation du schéma de protection des troupeaux en alpage : coût, travail, impact. In : Loup - Élevage : s'ouvrir à la complexité. Actes du séminaire des 15 et 16 juin 2006. Garde L. (Coord)., CERPAM, Manosque, France, 166-178.

Smith M.E., Linnell J., Odden J., Swenson J.E., 2010. Review of methods to reduce livestock depradation: I. Guardian animals. Acta Agric. Scandinavica, Section A - Anim. Sci., 50, 279-290

Templer V.L., Hampton R.R., 2013. Episodic memory in nonhuman animals. Current Biol., 23, R801-R806.

Trouwborst A., 2010. Managing the carnivore comeback: international and EU species protection Law and the return of Lynx, Wolf and Bear to Western Europe. J. Envir. Law, 22, 347-372.

Trouwborst A., Boitani L., Linnell J.D.C., 2017. Interpreting 'favourable conservation status' for large carnivores in Europe: how many are needed and how many are wanted? Biodiv. Conserv., 26, 37-61.

U.S. Fish and Wildlife Service, 2016. Northern Rocky Mountain Wolf Recovery Program 2015 Interagency Annual Report - Executive summary and tables. Helena, Montana, USA: 12p.

Vincent M., 2011. Les alpages à l'épreuve des loups. Quae \& MSH, Coll. Natures sociales, Paris, 352p. 
Wick P., 2003. Audition. In : Rapport fait au nom de la Commission d'enquête sur les conditions de la présence du loup en France et l'exercice du pastoralisme dans les zones de Montagnes. Estrosi C., Spagnou D. (Eds)., Assemblée Nationale, Rapport n 825 , Tome II, $1,88$.
Williams B.K., Brown E.D., 2014. Adaptive management: from more talk to real action. Envir. Manage., 53, 465-479.

\title{
Résumé
}

Vingt-cinq ans après leur arrivée en France, les loups sont régulièrement ou épisodiquement présents dans plus de 30 départements, avec 57 zones de présence permanente. Depuis 2008, la progression interannuelle des animaux d'élevage retrouvés prédatés (ovins, caprins, bovins, équins...) est linaire, avoisinant les 12000 en 2017. Il faut y ajouter les animaux disparus et les dégâts indirects : stress à effet durable, avortements, blessures internes et baisse de fertilité. La France a pourtant mis en œuvre et généralisé depuis 2004 une protection élaborée des troupeaux : présence humaine renforcée, chiens de protection et parcs de nuit électrifiés. Comment expliquer alors cet échec ? Il y a deux principales raisons. D'abord, les paysages composés de prairies, pelouses, haies, lisières et sous-bois, favorisent la prédation. Mais surtout, les loups se sont adaptés. Très intelligents et opportunistes, ils profitent en France de leur statut légal de protection stricte, n'associant visiblement plus les troupeaux aux humains et les humains au danger. Face à des troupeaux regroupés en parc de nuit, ils opèrent la moitié de leurs attaques en cours de journée, y compris en présence de chiens et d'humains. Dans les pays où les humains sont autorisés à défendre les troupeaux activement et promptement, y compris par des tirs létaux, les loups se tiennent plus à distance et les pertes d'élevage sont bien moindres. Sans une régulation ciblée des loups en fonction de leur comportement vis-à-vis des troupeaux, c'est la viabilité de nos élevages de plein air qui est profondément remise en question.

\begin{abstract}
Livestock farming and wolves in France: history, situation appraisal and solution pathway

Nowadays wolves are present on a regular or episodic basis in about a third of the country. Since 2008, yearly livestock killing due to wolves is growing linearly, and reached 12000 in 2017. This figure accounts neither for missing animals nor indirect damage: stress, abortions, fertility drops, etc. Since 2004, France has, however, implemented a now widespread set of protective measures: reinforced human presence, protection dogs and electrified night pens. The failure is clear, public and private costs are exploding. Wolf recovery goes on into fragmented landscapes of cultivated or natural meadows, hedges, edges and forest undergrowth, where grazing livestock is an easy prey. But mostly, wolves are smart and opportunistic. As strictly protected species, wolves need no care that livestock could be associated with humans and humans with danger. Half of the attacks now take place during the daytime, notwithstanding dogs and humans being present. Countries where humans are allowed a quick active and prompt defence of their livestock, including lethal shooting, give evidence of wolves keeping their distance and damaging much less. Without local and targeted regulation of wolves becoming prone to prey on livestock, the livelihood of farming practicing grazing in France is deeply jeopardized.
\end{abstract}

MEURET M., GARDE L., MOULIN C.-H., NOZIÈRES-PETIT M.-O., VINCENT M., 2017. Élevage et loups en France : historique, bilan et pistes de solution. INRA Prod. Anim., 30, 465-478. 